\title{
Coupled modelling of flood due to natural landslide dam breach
}

Wei Huang MSc

PhD candidate, State Key Laboratory of Water Resources and Hydropower Engineering Science, Wuhan University, Wuhan, China

Zhixian Cao PhD

Professor, State Key Laboratory of Water Resources and Hydropower Engineering Science, Wuhan University, China; Professor, Institute for Infrastructure and Environment, Heriot-Watt University, Edinburgh, UK Zhiyuan Yue PhD

Senior Engineer, Changjiang Waterway Planning, Design and Research Institute, Wuhan, China

\section{Gareth Pender PhD}

Professor, Institute for Infrastructure and Environment, Heriot-Watt University, UK; Visiting Professor, State Key Laboratory of Water Resources and Hydropower Engineering Science, Wuhan University, Wuhan, China

Jifu Zhou PhD

Professor, Institute of Mechanics, Chinese Academy of Science, Beijing, China

A coupled two-dimensional mathematical model is presented for a flood due to natural landslide dam breach. A new numerical algorithm is developed, preserving the well-balanced property between the flux and source terms of the momentum conservation equations over a mobile bed. It allows for the wetting-drying transition to be properly resolved. Equally importantly, the spatially variable size of the sediments of natural landslide dams is taken into account in estimating bed sediment entrainment flux. The model is first tested against flume experiments and then applied to a case study of the Tangjiashan landslide dam, the largest of its kind generated by the 2008 Wenchuan earthquake in Sichuan Province, China. Compared with field observations, the widening and deepening of the breach along with the flood hydrographs are reproduced fairly well by the present model as the heterogeneity of the dam material is incorporated. In contrast, when the heterogeneous composition of the natural landslide dam is ignored using a fixed sediment size, the model fails even if the parameters related to roughness, entrainment flux and mass collapse are largely tuned. An appropriate account of the heterogeneous composition of natural dams is therefore significant.

\section{Notation}

$c_{\mathrm{e}}$

$D$

$D_{x}$

d

E

$\boldsymbol{F}, \boldsymbol{G}$

$F_{x}$

g

h

n

$p_{0}$

$q_{\mathrm{b}}$

$q_{x}, q_{y}, q_{c}$

$\boldsymbol{S}$

$\boldsymbol{S}_{\mathrm{s}}, \boldsymbol{S}_{\mathrm{f}}$

$S_{\mathrm{R}}, S_{\mathrm{c}}$

$s \quad$ specific gravity of sediment

$\boldsymbol{U} \quad$ vector of conserved variables

$u, v \quad$ velocity components in $x$ - and $y$-directions

respectively

$\begin{array}{ll}z & \text { bed elevation } \\ \alpha & \text { empirical parameter }\end{array}$
$\Delta t \quad$ time step

$\Delta x, \Delta y \quad$ spatial steps in $x$ - and $y$-directions respectively

$\eta \quad$ water surface level above the datum

$\theta \quad$ Shields parameter

$\theta_{\mathrm{c}} \quad$ threshold Shields parameter for initiation of sediment movement

$\rho, \rho_{0} \quad$ densities of water-sediment mixture and saturated bed material

$\rho_{\mathrm{w}}, \rho_{\mathrm{s}} \quad$ densities of water and sediment respectively

$\tau_{\mathrm{b} x}, \tau_{\mathrm{b} y} \quad$ bed shear stresses

$\phi \quad$ modification coefficient

$\omega \quad$ settling velocity of a single sediment particle in tranquil clear water

\section{Introduction}

Landslide dams are often generated during earthquakes and/or heavy rainfall in storm-prone mountain areas, and may fail by overtopping and/or piping (Baker et al., 1993; Chen et al., 1992; Costa and Schuster, 1988; Cruden et al., 1997; Dai et al., 2005; Korup, 2002; Shang et al., 2003). The phenomenon occurs worldwide and flooding due to landslide dam failure can be catastrophic, leading to loss of life and property damage. Increasing attention has been paid to this phenomenon by governments, agencies and communities, but effective forecasting technologies are urgently needed to address this aspect of flood risk management. 
Over the last few decades, researchers have strived to enhance understanding of the mechanisms of landslide dam failure and the hydraulics of the resulting floods (Cao et al., 2011c). In particular, the breaching process has received considerable attention as it dictates the generation and propagation of the downstream flood wave and subsequent inundation, and is the most challenging process to model. As sufficient computing resources become available, physically based models are preferred for resolving the discharge hydrograph, stage hydrograph and the development of breaching without predefining or constraining the growth process. Wang and Bowles (2006a, 2006b) developed a physically based numerical model to simulate the breaching process of a non-cohesive dam. In their model, the two-dimensional (2D) shallow water equations were solved within the breach area to calculate the flow velocity and water depth. The erosion of the dam was calculated in three stages and the 3D slope stability was checked during the calculation. The model was decoupled - the influences of bed evolution on the flood flow were not fully represented. The erosion rate was used to calculate the bed change while the deposition in the breach area was not considered. Cao et al. (2011b, 2011c) developed a 2D coupled model to simulate landslide dam breach for both single and cascade dams composed of single-size sediment. In this model, the entire dam breaching process was modelled, which included the stage rise in the reservoir due to inflow, the breaching process of the landslide dam and the flood propagation downstream of the dam. A thorough review of landslide dam breaching models can be found in the literature (ASCE/EWRI, 2011).

The Tangjiashan landslide dam is the largest of the tens of large landslide dams induced by the 2008 Wenchuan earthquake in Sichuan Province, China (Cui et al., 2009). The potential failure of this huge dam placed more than 1.3 million people living in the downstream catchment at risk of catastrophic flooding. To support engineering decisions on managing the risk posed by the dam, a volume of field data has been collected (Liu et al., 2010), including data on the initial topography and composition of the dam, the geometry of the breach in its initial state and during the development of widening and deepening, as well as the discharge and water level at the breach. This volume of field data provides a unique opportunity to test computational model performance and capability. This field case has been studied before, as outlined below.

- Mori et al. (2010) proposed a two-layer model to simulate the breaching process of Tangjiashan landslide dam. The upper layer of their model was assumed to be clear water, while the lower layer was assumed to be a gravel-bed transport layer with a constant sediment concentration. The medium sediment size of the dam material was specified to be $0.5 \mathrm{~m}$, which was unreasonably large compared with $8 \mathrm{~mm}$ from field sampling (Liu et al., 2010).

- Chang and Zhang (2010) developed a 1D geotechnical model to resolve the breaching process. In this model, the heterogeneity of the dam material was taken into account with varied soil erodibility along the vertical. The crosssections of the breach were assumed to be trapezium shaped and a simplified weir equation was used to estimate the flow discharge through the breach. In this model, the hydrodynamics of the phenomenon were largely ignored: at least, the timing of the onset of the breach could not be resolved as the inflow from the far upstream was not incorporated at all.

- Wang et al. (2008a, 2008b) deployed both 1D and 2D models to simulate the breach development and resulting flood. The results of the $1 \mathrm{D}$ model were used to inform the emergency action taken by the flood defence agencies. The 2D model assumed a sediment transport capacity status, which may not be justified in dam break flows over erodible beds (Cao et al., 2004). Also, a single sediment size was adopted, ignoring the significant heterogeneity of the dam material. The model set-up also exhibited issues when the modelling results were compared against observed data. The initial stage was set about $2 \mathrm{~m}$ higher than the observed data (see Figure 6 in Wang et al., 2008b), rendering the computed discharge and stage hydrographs considerably higher than the observations.

To date, simplifications and assumptions have been introduced in previous modelling studies of not only the Tangjiashan landslide dam but others as well, whereas their impacts on the results have so far remained poorly understood. This inevitably renders the agreement between modelling and observations open to question. Further study is therefore warranted for physically enhanced modelling.

The paper presents a computational model for solving flooding due to landslide dam failure by overtopping. As an application of the model, a case study of the Tangjiashan landslide dam failure is presented. The model is extended from the recent coupled 2D mathematical model of Yue (2010) and Cao et al. (2011b). A new numerical algorithm is developed to deal with the source terms over a mobile bed, which properly resolves the wettingdrying transition and avoids non-physical velocity. Equally importantly, the spatially variable size of the sediments comprising the natural landslide dam is taken into account in estimating bed sediment entrainment flux, which comprises a step forward compared with previous studies. This model is first validated in two computational tests involving a static water basin and laboratory experiments of landslide dam failure by overtopping. It is then applied to study the breaching process of the Tangjiashan landslide dam and the resulting flood. A series of computational tests are conducted to address the sensitivity of the flood characteristics to the empirical parameters involved in estimations of boundary resistance and sediment entrainment.

\section{Mathematical model}

\subsection{Governing equations}

The governing equations are essentially the $2 \mathrm{D}$ shallow water equations comprising the complete mass and momentum conser- 
vation equations for the water-sediment mixture flow and the mass conservation equations for sediment and bed material respectively (Cao et al., 2011b; Yue, 2010). The governing equations can be written in a well-balanced conservative form as

1. $\frac{\partial \boldsymbol{U}}{\partial t}+\frac{\partial \boldsymbol{F}}{\partial x}+\frac{\partial \boldsymbol{G}}{\partial y}=\boldsymbol{S}$

$$
\boldsymbol{U}=\left[\begin{array}{c}
\eta \\
q_{x} \\
q_{y} \\
q_{c}
\end{array}\right]=\left[\begin{array}{c}
\eta \\
h u \\
h v \\
h c
\end{array}\right]
$$

$2 \mathrm{~b}$.

$$
\boldsymbol{F}=\left[\begin{array}{c}
h u \\
h u^{2}+0 \cdot 5 \boldsymbol{g}\left(\eta^{2}-2 \eta z\right) \\
h u v \\
h u c
\end{array}\right]
$$

$$
\text { 2c. } \quad \boldsymbol{G}=\left[\begin{array}{c}
h v \\
h u v \\
h v^{2}+0 \cdot 5 \boldsymbol{g}\left(\eta^{2}-2 \eta z\right) \\
h v c
\end{array}\right]
$$

$$
\boldsymbol{S}_{=} \boldsymbol{S}_{\mathrm{s}}+\boldsymbol{S}_{\mathrm{f}}=\left[\begin{array}{c}
0 \\
-\boldsymbol{g} \eta \frac{\partial z}{\partial x} \\
-\boldsymbol{g} \eta \frac{\partial z}{\partial y} \\
0
\end{array}\right]
$$

$$
+\left[\begin{array}{c}
0 \\
-\frac{\tau_{\mathrm{b} x}}{\rho}-\frac{\left(\rho_{\mathrm{s}}-\rho_{\mathrm{w}}\right) \boldsymbol{g} h^{2}}{2 \rho} \frac{\partial c}{\partial x}-\frac{\left(\rho_{0}-\rho\right)(E-D) u}{\rho\left(1-p_{0}\right)} \\
-\frac{\tau_{\mathrm{b} y}}{\rho}-\frac{\left(\rho_{\mathrm{s}}-\rho_{\mathrm{w}}\right) \boldsymbol{g} h^{2}}{2 \rho} \frac{\partial c}{\partial y}-\frac{\left(\rho_{0}-\rho\right)(E-D) v}{\rho\left(1-p_{0}\right)} \\
E-D
\end{array}\right]
$$

3. $\frac{\partial z}{\partial t}=\frac{D-E}{1-p_{0}}$ where $\boldsymbol{U}$ is a vector of conserved variables, $\boldsymbol{F}$ and $\boldsymbol{G}$ are the convective flux vectors of the flow in the $x$ - and $y$-directions respectively, $\boldsymbol{S}$ is the source term including the bed slope $\boldsymbol{S}_{\mathrm{s}}$, friction source term $\boldsymbol{S}_{\mathrm{f}}$ and other terms related to sediment transport and bed evolution, $\eta$ is the water surface level above the datum, $z$ is the bed elevation (the water depth is then evaluated by $h=\eta-z), u$ and $v$ are velocity components in the $x$ - and $y$ directions respectively, $c$ is the depth-averaged volumetric sediment concentration, $\boldsymbol{g}$ is the gravitational acceleration, $p_{0}$ is the bed sediment porosity, $\rho_{\mathrm{w}}$ and $\rho_{\mathrm{s}}$ are the densities of water and sediment respectively, $\rho$ and $\rho_{0}$ are the densities of the watersediment mixture and the saturated bed material where $\rho=\rho_{\mathrm{w}}(1-c)+\rho_{\mathrm{s}} c$ and $\rho_{0}=\rho_{\mathrm{w}} p_{0}+\rho_{\mathrm{s}}\left(1-p_{0}\right), E$ and $D$ are sediment entrainment and deposition fluxes across the bottom boundary of the flow, representing the sediment exchange between the water column and the river bed, $\tau_{\mathrm{b} x}$ and $\tau_{\mathrm{b} y}$ are bed friction stresses, and $q_{x}=h u, q_{y}=h v, q_{c}=h c$ are conservative variables. The pressure terms in the momentum conservation equations have been properly transformed for mathematical balance between the flux and source terms. For instance, in the $x$ direction, it is implicitly embedded in the terms $\partial\left[\boldsymbol{g}\left(\eta^{2}-2 \eta z\right) /\right.$ 2] $/ \partial x$ and $\boldsymbol{g} \eta \partial z / \partial x$.

\subsection{Model closure}

To close the governing equations, formulae have to be employed to determine the bed shear stress and the sediment exchange fluxes. In general, dam break floods are unsteady and nonuniform, featuring boundary resistance substantially different from those of steady and uniform flows. This fact is more pronounced as sediment transport is involved and the bed is rendered mobile and exhibits bedforms (e.g. dunes). Unfortunately, there is no generally applicable relationship representing boundary resistance in such flows. Consequently, computational studies of dam break floods over fixed and mobile beds have exclusively used resistance relationships initially developed for steady and uniform flows, which are usually based on Manning's equation involving the roughness parameter $n$. The present model follows the same practice in this regard

4a. $\tau_{\mathrm{b} x}=\rho \boldsymbol{g} n^{2} q_{x}\left(q_{x}^{2}+q_{y}^{2}\right)^{1 / 2} / h^{7 / 3}$

4b. $\quad \tau_{\mathrm{b} y}=\rho \boldsymbol{g} n^{2} q_{y}\left(q_{x}^{2}+q_{y}^{2}\right)^{1 / 2} / h^{7 / 3}$

Two distinct mechanisms are involved in the sediment exchange between the flow and bed; that is, sediment entrainment due to turbulence and particle-particle interactions, and sediment deposition mainly due to gravitational settling. Current understanding of these remains far from complete and, inevitably, quantification of sediment entrainment and deposition fluxes is largely empirical. Given this fact, it is sensible to estimate the fluxes using existing sediment transport formulae and carefully 
appreciate the uncertainty with a modification coefficient. Accordingly, the entrainment and deposition fluxes are estimated by

$$
\text { 5a. } E=\alpha \omega c_{\mathrm{e}}\left(1-c_{\mathrm{e}}\right)^{2}
$$

$$
\text { 5b. } \quad D=\alpha \omega c(1-\alpha c)^{2}
$$

where $\omega$ is the settling velocity of a single sediment particle in tranquil clear water, which is calculated using the formula of Zhang and Xie (1993). Here, the impact of sediment concentration on the settling velocity is considered (Richardson and Zaki, 1954). Bed load sediment transport capacity $c_{\mathrm{e}}$ is determined from the local flow regime. The empirical parameter $\alpha$ represents the difference between the near-bed concentration and the depthaveraged concentration; its value is here estimated using the method of Yue (2010) and Cao et al. (2011b). The bed load sediment transport capacity $c_{\mathrm{e}}$ is given by

6a. $c_{\mathrm{e}}=q_{\mathrm{b}} / h\left(u^{2}+v^{2}\right)^{1 / 2}$

6b. $\quad q_{\mathrm{b}}=\phi 8\left(s \boldsymbol{g} d^{3}\right)^{1 / 2}\left(\theta-\theta_{\mathrm{c}}\right)^{3 / 2}$

where $q_{\mathrm{b}}$ is the unit-width bed load transport rate at transport capacity status, $s$ is the specific gravity of sediment, $d$ is the medium diameter of the local sediment, $\theta$ is the Shields parameter and $\theta_{\mathrm{c}}$ is the threshold Shields parameter for initiation of sediment movement, determined following Yue (2010). There has been a plethora of empirical formulations for $q_{\mathrm{b}}$. Yet existing formulations for bed load transport rate are derived under steady and uniform flows, and therefore the entrainment flux based on these formulations may not be directly applicable to dam breach flood flows. A slightly modified version of the MPM (Meyer-Peter and Müller, 1948) formula is introduced here, with a modification coefficient $\phi$ to be calibrated using measured data. This is justified because the Shields parameter in dam break floods can go beyond the range in which the MPM formula was initially derived. In essence, it is a functional form of bed load transport rate introduced based on the MPM formula. In the present study, this parameter is one of the major sources of uncertainty and its influence on the results is analysed in a later section.

Theoretically, a 3D model could be incorporated to represent mass collapse (e.g. Wang and Bowles, 2006a, 2006b). However, it is too complicated and computationally costly to be applicable to field cases at the present time. Furthermore, the application of a 3D model is constrained by the unavailability of detailed data of landslide dam composition. A purely geometric approach ignores some details of mass collapse from a geotechnical perspective, such as the stress profile, the sliding process and the settlement of mass collapse. And yet, it is easy to use and in recent years has been found to be able to reasonably model the lateral collapse. Therefore it is adopted in the present model to account for the mass collapse, as described in Yue (2010) and Cao et al. (2011b). Two slopes are involved - the slope in line with the repose angle of sediment $\left(S_{\mathrm{R}}\right)$ and a critical slope for bank instability $\left(S_{\mathrm{c}}\right)$.

\subsection{Incorporating heterogeneous composition of landslide dams}

Natural landslide dams are always composed of heterogeneous sediments (Cencetti et al., 2006; Chang and Zhang, 2010) with sizes widely distributed. Commonly, previous mathematical models deal with natural dam material with a fixed-size approach (i.e. the sediment size is kept at a single value, normally the medium sediment diameter, throughout the modelling). Although sometimes they can approximately reproduce the peak discharge, the breaching process, including its initiation and termination, may not be adequately predicted. The model of Yue (2010) and Cao et al. (2011b) was initially developed for experimental landslide dams in the laboratory, of which sediment size is not as widely distributed as in natural dams. Here, the model is extended to incorporate graded sediments, which is a step forward for the modelling of a natural landslide dam breach.

Succinctly, a complete graded sediment model (Wu, 2007; Wu et al., 2000) is needed, which involves numerical solution of a number of continuity equations of sediments (one for each sediment size). Inevitably, it is computationally expensive. Furthermore, implementing a complete graded sediment transport model would require sufficiently detailed data of the landslide dam composition and the river bed in question, which is mostly unavailable for a field case. Here an approach 'compromised' between computational costs, limited data availability and representation of physical process is adopted. In this approach, only the continuity equation for the global sediment transport is solved, for which the estimation of sediment entrainment flux is the key to properly resolving the dam breach and the resulting flood as the deposition flux can be readily estimated using the local volumetric sediment concentration. The entrainment flux is estimated with respect to the local sediment size on the bed surface, which may vary in space. By means of this procedure, the heterogeneous nature of the sediments comprising the natural landslide dam is mostly captured. This approach is implemented only within the domain initially occupied by the landslide dam, while beyond the domain a fixed-size model is used because of the unavailability of detailed data on bed composition. This inevitably introduces inaccuracy and yet it is trivial as, over the dam, sediment entrainment is always overwhelming compared with deposition. In this respect, this approach is similar to that proposed by Chang and Zhang (2010), as it takes into account sediment erosion only and ignores the contribution from sediment deposition.

\subsection{Numerical scheme}

The numerical algorithm employed in the present mobile bed model is, in principle, an extension of that in the 1D model over 
a fixed bed (Liang and Marche, 2009). A finite-volume Godunovtype method incorporating the HLLC (Harten-Lax-van Leer contact wave) approximate Riemann solver is used to obtain the numerical solutions on a fixed rectangular mesh. In this regard, it is recognised that a rather fine mesh may have to be used for appropriate numerical accuracy, which inevitably increases the computing cost. This actually relates to a fundamental issue in the context of computational hydraulics; that is, the use of an adaptive mesh to achieve a sensible balance between computational accuracy and efficiency, which is certainly warranted in future studies.

The following time-marching formulae are used to update the flow and sediment variables to a new time step $(k+1)$

7. $\boldsymbol{U}_{i, j}^{p}=\boldsymbol{U}_{i, j}^{k}+\Delta t \boldsymbol{S}_{\mathrm{f} i, j}^{k+1}$

$$
\begin{aligned}
& \boldsymbol{U}_{i, j}^{k+1}=\boldsymbol{U}_{i, j}^{p}-\frac{\Delta t\left(\boldsymbol{F}_{i+1 / 2, j}-\boldsymbol{F}_{i-1 / 2, j}\right)^{p}}{\Delta x} \\
\text { 8. } & -\frac{\Delta t\left(\boldsymbol{G}_{i, j+1 / 2}-\boldsymbol{G}_{i, j-1 / 2}\right)^{p}}{\Delta y}+\Delta t \boldsymbol{S}_{\mathrm{s} i, j}^{k}
\end{aligned}
$$

in which the superscript $k$ represents the time level and the superscript $p$ indicates the state after calculating variables from Equation 7 , subscripts $(i, j)$ are the spatial node indexes, $\Delta t$ is the time step, $\Delta x$ and $\Delta y$ denote the spatial steps and $\boldsymbol{F}_{i+1 / 2, j}$, $\boldsymbol{F}_{i-1 / 2, j}, \boldsymbol{G}_{i, j+1 / 2}$ and $\boldsymbol{G}_{i, j-1 / 2}$ are interface fluxes.

In Equation 7, the friction source term $S_{\mathrm{f}}^{k+1}$ is solved by a splitting method; it is equivalent to solving the ordinary differential equations

$$
\text { 9. } \frac{d \boldsymbol{U}}{d t}=S_{f}
$$

Consider the momentum equation of Equation 9 in the $x$ direction

$$
\begin{aligned}
\frac{\mathrm{d} q_{x}}{\mathrm{~d} t}= & S_{\mathrm{f} x} \\
= & \frac{-\tau_{\mathrm{b} x}}{\rho}-\frac{\left(\rho_{\mathrm{s}}-\rho_{\mathrm{w}}\right) \boldsymbol{g} h^{2}}{2 \rho} \frac{\partial c}{\partial x} \\
10 . \quad & -\frac{\left(\rho_{0}-\rho\right)(E-D) q_{x}}{\rho\left(1-p_{0}\right) h}
\end{aligned}
$$

Equation 10 is then discretised by a full implicit method
11. $\frac{q_{x}^{p}-q_{x}^{k}}{\Delta t}=S_{\mathrm{f} x}^{k+1}$

where the friction source term $S_{\mathrm{f} x}^{k+1}$ is expressed using a Taylor series as

12. $S_{\mathrm{f} x}^{k+1}=S_{\mathrm{f} x}^{k}+\left(\partial S_{\mathrm{f} x} / \partial q_{x}\right)^{k} \Delta q_{x}+O\left(\Delta q_{x}^{2}\right)$

where $\Delta q_{x}=q_{x}^{p}-q_{x}^{k}$. Neglecting the high-order terms and substituting it into Equation 11, the following formula is attained for updating $q_{x}$ to the $p$ time step

13. $q_{x}^{p}=q_{x}^{k}+\Delta t\left(S_{\mathrm{f} x} / D_{x}\right)^{k}=q_{x}^{k}+\Delta t F_{x}$

where $D_{x}=1-\Delta t\left(\partial S_{\mathrm{f} x} / \partial q_{x}\right)^{k}$ is the implicit coefficient and $F_{x}=S_{\mathrm{f} x} / D_{x}$ is the friction source term including the implicit coefficient. In order to ensure stability, a limiting value of the implicit friction force must be identified according to the physics of the shallow flows. The maximum effect of the friction force is to constrain the flow (i.e. $q_{x}^{p} q_{x}^{k} \geqslant 0$ ). Then, according to Equation 13 , the limiting value of the implicit friction force can be easily derived as

14. $F_{x} \begin{cases}\geqslant-q_{x}^{k} / \Delta t & \text { if } q_{x}^{k} \geqslant 0 \\ \leqslant-q_{x}^{k} / \Delta t & \text { if } q_{x}^{k} \leqslant 0\end{cases}$

If $F_{x}$ computed is beyond the limit, its value is replaced by the critical value $F_{x}=-q_{x}^{k} / \Delta t$ in the actual computation. Similarly, the implicit coefficient in the $y$-direction can be calculated. In Equation 10 , the term $\partial c / \partial x$ is discretised using a central difference scheme for simplicity.

When evaluating $\boldsymbol{U}_{i, j}^{k+1}$ in Equation 8, the solution of Equation 7 is used as the initial condition. The interface fluxes $\boldsymbol{F}_{i+1 / 2, j}$, $\boldsymbol{F}_{i-1 / 2, j}, \boldsymbol{G}_{i, j+1 / 2}$ and $\boldsymbol{G}_{i, j-1 / 2}$ are computed using the HLLC Riemann solver (Toro, 2001), which needs correct reconstruction of the Riemann states. The MUSCL method is used to achieve second-order accuracy in space. The Riemann states are defined by using the minmod slope-limited reconstruction. At the lefthand side of the cell interface $(i+1 / 2, j)$, the values are evaluated by 


$$
\begin{aligned}
\bar{\eta}_{i+1 / 2, j}^{\mathrm{L}} & =\eta_{i, j}+0 \cdot 5 \psi\left(\eta_{i, j}-\eta_{i-1, j}\right) \\
\bar{h}_{i+1 / 2, j}^{\mathrm{L}} & =h_{i, j}+0 \cdot 5 \psi\left(h_{i, j}-h_{i-1, j}\right) \\
\bar{z}_{i+1 / 2, j}^{\mathrm{L}} & =\bar{\eta}_{i+1 / 2, j}^{\mathrm{L}}-\bar{h}_{i+1 / 2, j}^{\mathrm{L}} \\
\bar{q}_{x i+1 / 2, j}^{\mathrm{L}} & =q_{x i, j}+0 \cdot 5 \psi\left(q_{x i, j}-q_{x i-1, j}\right) \\
\bar{q}_{y i+1 / 2, j}^{\mathrm{L}} & =q_{y i, j}+0 \cdot 5 \psi\left(q_{y i, j}-q_{y i-1, j}\right) \\
15 . \quad \bar{q}_{c i+1 / 2, j}^{\mathrm{L}} & =q_{c i, j}+0 \cdot 5 \psi\left(q_{c i, j}-q_{c i-1, j}\right)
\end{aligned}
$$

where $\psi$ represents the slope-limited function evaluated at cell $(i, j)$ based on the flow and sediment data at the cell and its upwind and downwind neighbours, and the minmod slope limiter is used for better numerical stability (Hirsch, 1990)

\section{6. $\psi(r)=\max [0, \min (r, 1)]$}

where $r$ is the ratio of successive gradients of the flow and the variable under consideration; for example, for $\eta$

$$
\text { 17. } r=\frac{\eta_{i+1, j}-\eta_{i, j}}{\eta_{i, j}-\eta_{i-1, j}}
$$

Similar expressions can be defined for $q_{x}, q_{y}, q_{c}$ and $h$.

The interface values of the right-hand side of $(i+1 / 2, j)$ are calculated in a similar way.

$$
\begin{gathered}
\bar{\eta}_{i+1 / 2, j}^{\mathrm{R}}=\eta_{i+1, j}-0 \cdot 5 \psi\left(\eta_{i+1, j}-\eta_{i, j}\right) \\
\bar{h}_{i+1 / 2, j}^{\mathrm{R}}=h_{i+1, j}-0 \cdot 5 \psi\left(h_{i+1, j}-h_{i, j}\right) \\
\bar{z}_{i+1 / 2, j}^{\mathrm{R}}=\bar{\eta}_{i+1 / 2, j}^{\mathrm{R}}-\bar{h}_{i+1 / 2, j}^{\mathrm{R}} \\
\bar{q}_{x i+1 / 2, j}^{\mathrm{R}}=q_{x i+1, j}-0 \cdot 5 \psi\left(q_{x i+1, j}-q_{x i, j}\right) \\
\bar{q}_{y i+1 / 2, j}^{\mathrm{R}}=q_{y i+1, j}-0 \cdot 5 \psi\left(q_{y i+1, j}-q_{y i, j}\right)
\end{gathered}
$$

18. $\bar{q}_{c i+1 / 2, j}^{\mathrm{R}}=q_{c i+1, j}-0 \cdot 5 \psi\left(q_{c i+1, j}-q_{c i, j}\right)$

where $\psi$ is evaluated at cell $(i+1, j)$. The velocity components and volumetric sediment concentration are then calculated by

$$
\begin{aligned}
\bar{u}_{i+1 / 2, j}^{\mathrm{L}} & =\bar{q}_{x i+1 / 2, j}^{\mathrm{L}} / \bar{h}_{x i+1 / 2, j}^{\mathrm{L}} \\
\bar{u}_{i+1 / 2, j}^{\mathrm{R}} & =\bar{q}_{x i+1 / 2, j}^{\mathrm{R}} / \bar{h}_{x i+1 / 2, j}^{\mathrm{R}} \\
\bar{v}_{i+1 / 2, j}^{\mathrm{L}} & =\bar{q}_{y i+1 / 2, j}^{\mathrm{L}} / \bar{h}_{y i+1 / 2, j}^{\mathrm{L}} \\
\bar{v}_{i+1 / 2, j}^{\mathrm{R}} & =q_{y i+1 / 2, j}^{\mathrm{R}} / \bar{h}_{y i+1 / 2, j}^{\mathrm{R}} \\
\bar{c}_{i+1 / 2, j}^{\mathrm{L}} & =\bar{q}_{c i+1 / 2, j}^{\mathrm{L}} / \bar{h}_{x i+1 / 2, j}^{\mathrm{L}} \\
19 . \quad \bar{c}_{i+1 / 2, j}^{\mathrm{R}} & =\bar{q}_{c i+1 / 2, j}^{\mathrm{R}} / \bar{h}_{x i+1 / 2, j}^{\mathrm{R}}
\end{aligned}
$$

Based on the above interface values, the Riemann states can be sought for designing the non-negativity of water depth. As suggested by Liang and Marche (2009), a single bed elevation at the cell interface $(i+1 / 2, j)$ may be defined as

$$
z_{i+1 / 2, j}=\max \left(\bar{z}_{i+1 / 2, j}^{\mathrm{L}}, \bar{z}_{i+1 / 2, j}^{\mathrm{R}}\right)
$$

The depth components of the Riemann states are then defined by

$$
\begin{aligned}
& h_{i+1 / 2, j}^{\mathrm{L}}=\max \left(0, \bar{\eta}_{i+1 / 2, j}^{\mathrm{L}}-z_{i+1 / 2, j}\right) \\
\text { 20. } \quad h_{i+1 / 2, j}^{\mathrm{R}} & =\max \left(0, \bar{\eta}_{i+1 / 2, j}^{\mathrm{R}}-z_{i+1 / 2, j}\right)
\end{aligned}
$$

which preserves positive water depth. The Riemann states of other flow variables can be obtained accordingly

$$
\begin{aligned}
\eta_{i+1 / 2, j}^{\mathrm{L}} & =h_{i+1 / 2, j}^{\mathrm{L}}+z_{i+1 / 2, j} \\
q_{x i+1 / 2, j}^{\mathrm{L}} & =\bar{u}_{i+1 / 2, j}^{\mathrm{L}} h_{i+1 / 2, j}^{\mathrm{L}} \\
q_{y i+1 / 2, j}^{\mathrm{L}} & =\bar{v}_{i+1 / 2, j}^{\mathrm{L}} h_{i+1 / 2, j}^{\mathrm{L}} \\
q_{c i+1 / 2, j}^{\mathrm{L}} & =\bar{c}_{i+1 / 2, j}^{\mathrm{L}} h_{i+1 / 2, j}^{\mathrm{L}} \\
\eta_{i+1 / 2, j}^{\mathrm{R}} & =h_{i+1 / 2, j}^{\mathrm{R}}+z_{i+1 / 2, j} \\
q_{x i+1 / 2, j}^{\mathrm{R}} & =\bar{u}_{i+1 / 2, j}^{\mathrm{R}} h_{i+1 / 2, j}^{\mathrm{R}} \\
q_{y i+1 / 2, j}^{\mathrm{R}} & =\bar{v}_{i+1 / 2, j}^{\mathrm{R}} h_{i+1 / 2, j}^{\mathrm{R}} \\
q_{c i+1 / 2, j}^{\mathrm{R}} & =\bar{c}_{i+1 / 2, j}^{\mathrm{R}} h_{i+1 / 2, j}^{\mathrm{R}}
\end{aligned}
$$

The bed slope term $\boldsymbol{S}_{\mathrm{s}}^{k}$ is discretised using the method proposed by Liang and Marche (2009). The procedure for the $x$-direction is outlined below; that for the $y$-direction is similar. For the dry bed case, a numerical technique needs to be employed in order to preserve a well-balanced solution 
22. $S_{\mathrm{s} x}=-\boldsymbol{g} \eta \frac{\partial z}{\partial x}+S_{0 i-1 / 2, j}+S_{0 i+1 / 2, j}$

The first term on the right-hand side is discretised as

23. $-\boldsymbol{g} \eta \frac{\partial z}{\partial x}=-\boldsymbol{g} \bar{\eta}\left(\frac{z_{i+1 / 2, j}-z_{i-1 / 2, j}}{\Delta x}\right)$

where

$$
\bar{\eta}=\left(\eta_{i-1 / 2, j}^{\mathrm{R}}+\eta_{i+1 / 2, j}^{\mathrm{L}}\right) / 2
$$

The other two terms are evaluated from

24a. $S_{0 i-1 / 2, j}=\boldsymbol{g} \Delta z_{i-1 / 2, j} \frac{z_{i+1 / 2, j}-\left(z_{i-1 / 2, j}-\Delta z_{i-1 / 2, j}\right)}{2 \Delta x}$

24b. $S_{0 i+1 / 2, j}=\boldsymbol{g} \Delta z_{i+1 / 2, j} \frac{\left(z_{i+1 / 2, j}-\Delta z_{i+1 / 2, j}\right)-z_{i-1 / 2, j}}{2 \Delta x}$

where

$$
\begin{aligned}
\Delta z_{i-1 / 2} & =\max \left[0,-\left(\bar{\eta}_{i-1 / 2, j}^{\mathrm{R}}-z_{i-1 / 2, j}\right)\right] \\
\text { 25. } \Delta z_{i+1 / 2} & =\max \left[0,-\left(\bar{\eta}_{i+1 / 2, j}^{\mathrm{L}}-z_{i+1 / 2, j}\right)\right]
\end{aligned}
$$

Bed evolution is computed explicitly from Equation 3, and is written as

26. $z_{i, j}^{k+1}=z_{i, j}^{k}+\Delta t(D-E)_{i, j}^{k} /\left(1-p_{0}\right)$

The current numerical scheme is explicit and its stability is controlled by the Courant-Friedrichs-Lewy condition. The time step is given by

$$
\Delta t=\operatorname{Crmin}\left(\frac{\Delta x}{\left|u_{i, j}\right|+\left(\boldsymbol{g} h_{i, j}\right)^{1 / 2}}, \frac{\Delta y}{\left|v_{i, j}\right|+\left(\boldsymbol{g} h_{i, j}\right)^{1 / 2}}\right)
$$

where $\mathrm{Cr}$ is the Courant number. For all the cases in the present work, it is specified as $\mathrm{Cr}=0 \cdot 4$. Furthermore, double precision is adopted throughout the computation.

\subsection{Computational tests}

To test the mathematical balance between the flux and source terms (i.e. well-balanced property) of the present model, a static water basin is designed based on the topography proposed by Brufau et al. (2002). The topography was fixed in the work of Brufau et al. (2002), but it is modified here to be mobile and is composed of uniform sand of diameter $0.01 \mathrm{~mm}$. The height of the wall of the basin is $2.0 \mathrm{~m}$, and a hump is located in the basin, of which the bed elevation is defined by

$$
z(x, y)=\max \left(0,2 \cdot 5-0 \cdot 3\left[(x-47 \cdot 5)^{2}+(y-15)^{2}\right]^{1 / 2}\right)
$$

At the initial time, the water is static. No water or sediment flows in or out of the basin (i.e. the water and sediment fluxes across the walls vanish during the computation). Figure 1 shows the stage, velocity components and volumetric sediment concentration at $t=300 \mathrm{~s}$. It is shown that the water level remains unchanged compared with its initial static state (Figure 1(a)). The velocity components in both $x$-and $y$-directions are preserved as $0.0 \mathrm{~m} / \mathrm{s}$ - that is, no spurious velocity is generated by the present model (Figures $1(\mathrm{~b})$ and $1(\mathrm{c})$ ) to the accuracy of $1.0 \times 10^{-12} \mathrm{~m} / \mathrm{s}$ - and, in line with this, sediment concentration is preserved as 0.0 to the accuracy of $1.0 \times 10^{-12}$ (i.e. no spurious sand is entrained into the water) (Figure 1(d)). This test also indicates that the present model can appropriately deal with the irregular topography and the wetting-drying transition. In fact, the wellbalanced property is independent of the numerical scheme being used to calculate the flux and the bed slope source term, which has been strictly proved for both the wet and dry cases (Liang and Marche, 2009).

Yue (2010) and Cao et al. (2011a) documented a series of flume experiments on dam breach and the resulting floods. These experiments were carried out in a large-scale flume of $80 \mathrm{~m} \times$ $1.2 \mathrm{~m} \times 0.8 \mathrm{~m}$; the bed slope of the flume was 0.001 and Manning's roughness was approximately $0 \cdot 012$. A set of 12 automatic water-level probes was used to measure the stage hydrographs at 12 cross-sections along the flume. The tracking speed of the water-level probes was set at $100 \mathrm{~mm} / \mathrm{s}$; the corresponding measurement error was within $\pm 0.5 \mathrm{~mm}$ at the sampling frequency of $2 \mathrm{~Hz}$. Different discharges from the upstream, the initial dam height, dam material composition, upstream and downstream slopes of the dam surface and initial breach dimensions were tested in the study. In the experiments, the dam failed by virtue of erosion of the overtopping flow and, when there was an initial breach, lateral collapse was also observed. The experiments provided a unique and systematic set of observed data for testing mathematical models of dam breach and the resulting flood.

To demonstrate the performance of the present model, two experimental cases using nearly uniform sands are revisited: Fcase 11 and S-case 7 with and without initial dam breach respectively (Cao et al., 2011a, 2011b). In both cases, the initial upstream and downstream slopes of the dam are $1 / 2$ and $1 / 3$ respectively. The medium diameter of the non-cohesive dam material is $0.8 \mathrm{~mm}$ and the specific gravity of the sediment is 


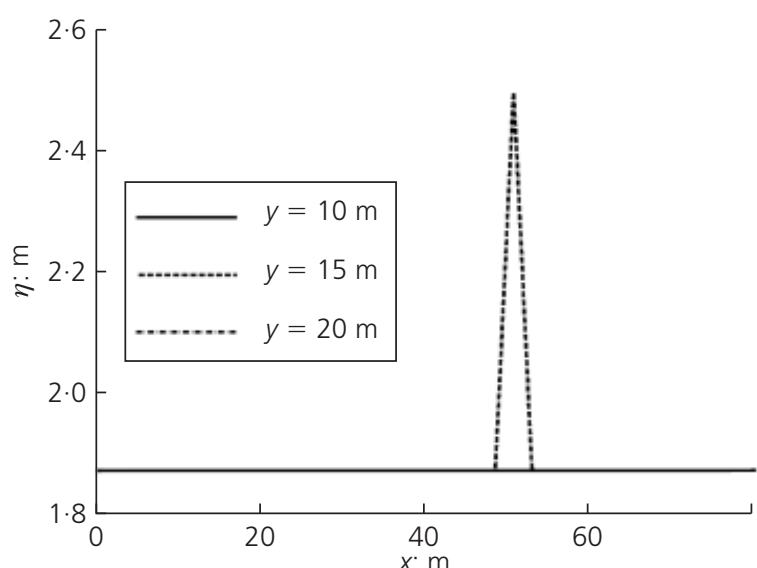

(a)

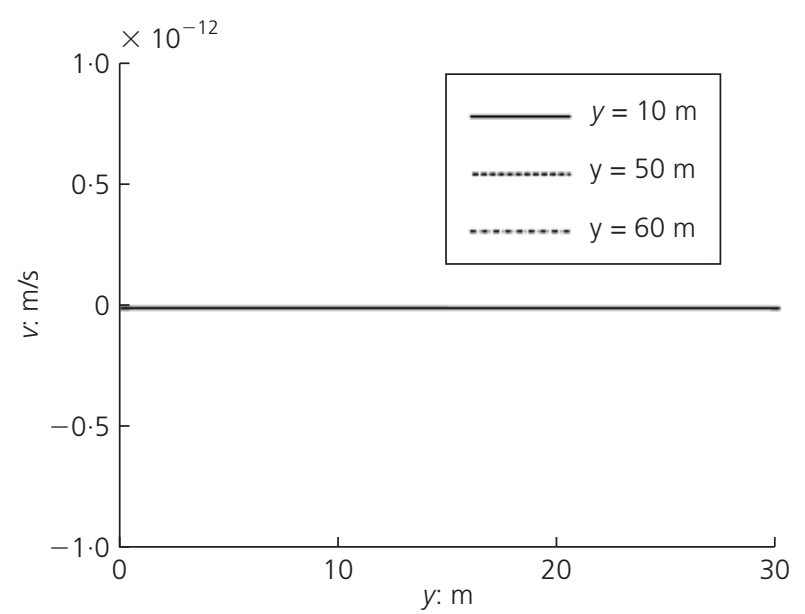

(c)

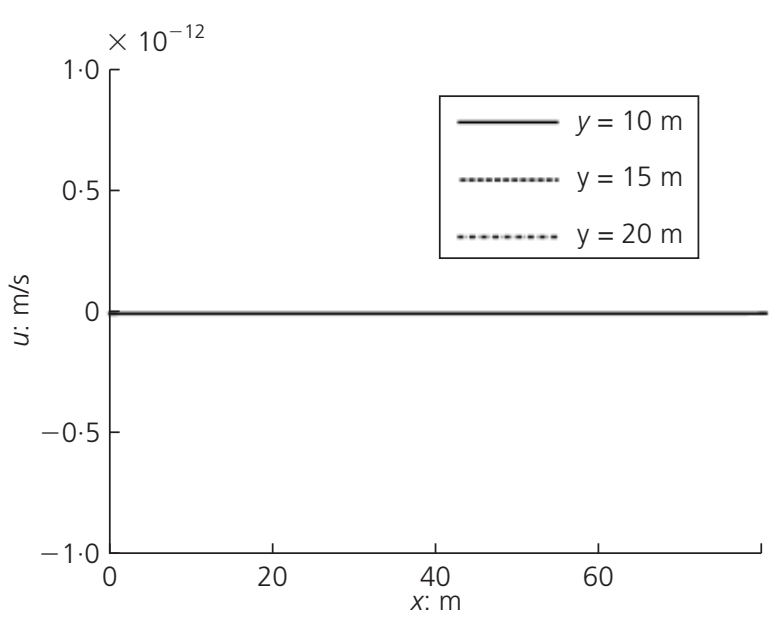

(b)

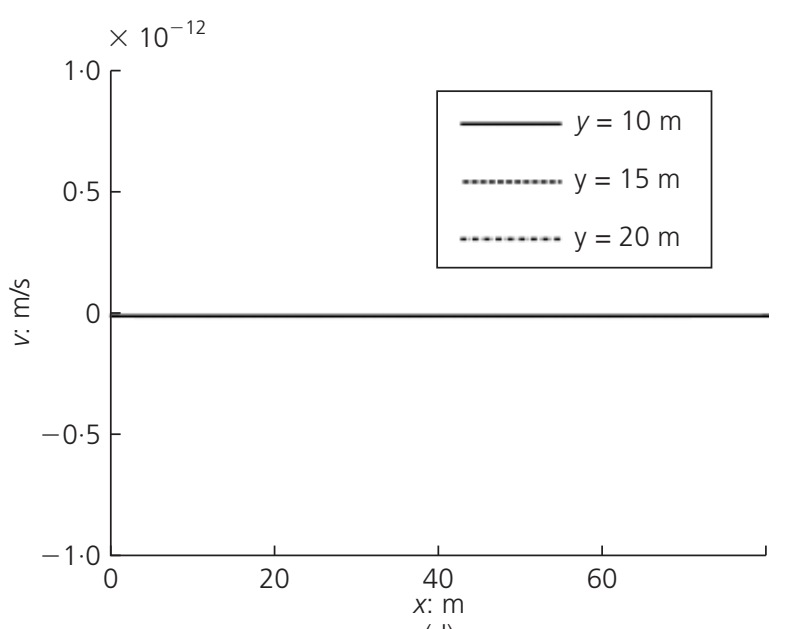

(d)

Figure 1. Computed results of static water case at $t=300 \mathrm{~s}$

1.65. The critical slope calibrated to $2 \cdot 0$ by Cao et al. (2011b). The inlet discharge is $0.042 \mathrm{~m}^{3} / \mathrm{s}$ for both cases. For S-case 7 , the initial static water depths immediately upstream and downstream of the dam are $0.051 \mathrm{~m}$ and $0.046 \mathrm{~m}$ respectively. The initial dam height is $0.4 \mathrm{~m}$ with a $0.1 \mathrm{~m}$ deep initial breach. For F-case 11, the initial static water depths immediately upstream and downstream of the dam are $0.054 \mathrm{~m}$ and $0.048 \mathrm{~m}$ respectively.

Figures 2 and 3 show a comparison of the computed and measured stage hydrographs at four cross-sections: CS1 and CS5 (respectively $22 \mathrm{~m}$ and $1 \mathrm{~m}$ upstream of the dam) and CS8 and CS12 (respectively $13 \mathrm{~m}$ and $32.5 \mathrm{~m}$ downstream of the dam). Figure 2 shows the results for the dam without an initial breach; the computed stages are nearly in line with those measured, especially at the descending phase. Figure 3 shows the results for the dam with an initial breach, and the computed stages agree well with those measured. Overall, the computed stage hydrographs are in satisfactory agreement with the measured data for both cases.
Figure 4 illustrates the development of the dam breach and the temporal variation of the velocity distribution for S-case 7 featuring an initial breach. At $t=280 \mathrm{~s}$, the water flows through the breach and reaches the toe of the dam. The downstream surface of the dam therefore begins to erode. The eroded sediments deposit around the toe of the dam. Compared with that at $t=280 \mathrm{~s}$, the breach at $t=380 \mathrm{~s}$ is wider and deeper. At this moment, the velocities downstream of the dam are distributed over this wider area. At $t=400 \mathrm{~s}$, the breach is further widened and deeper than that at $t=380 \mathrm{~s}$. Only a small part of the dam remains near the flume walls. The velocities have spread further than those at $t=380 \mathrm{~s}$. At $t=600 \mathrm{~s}$, the dam area is totally eroded and the velocities are nearly parallel to the flume side walls. In Figure 4, backward erosion is clearly shown by the dam topography at $t=280 \mathrm{~s}, 380 \mathrm{~s}$ and $400 \mathrm{~s}$. The figure also shows that no spurious velocity is generated during the dam breach process, which is typically characterised by rapid wetting-drying transition. 
Coupled modelling of flood due to

natural landslide dam breach

Huang, Cao, Yue, Pender and Zhou

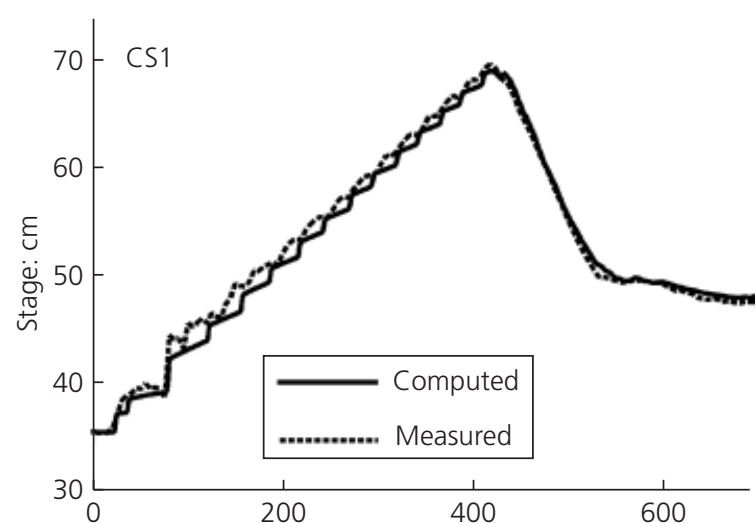

(a)

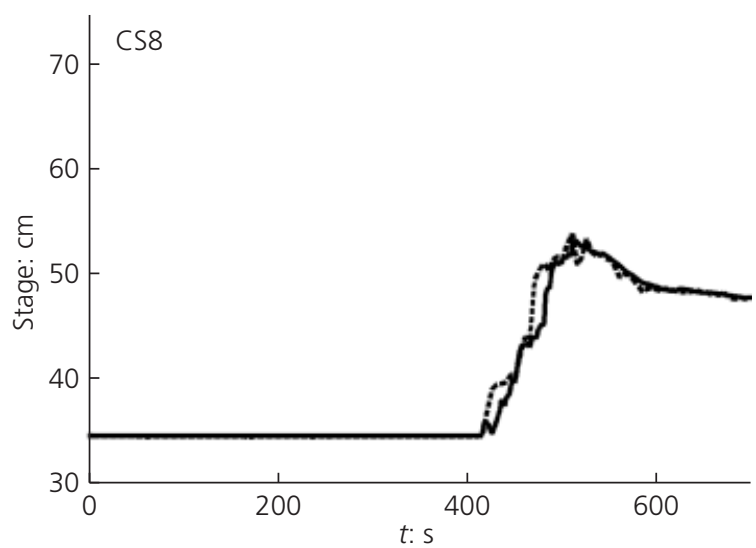

(c)

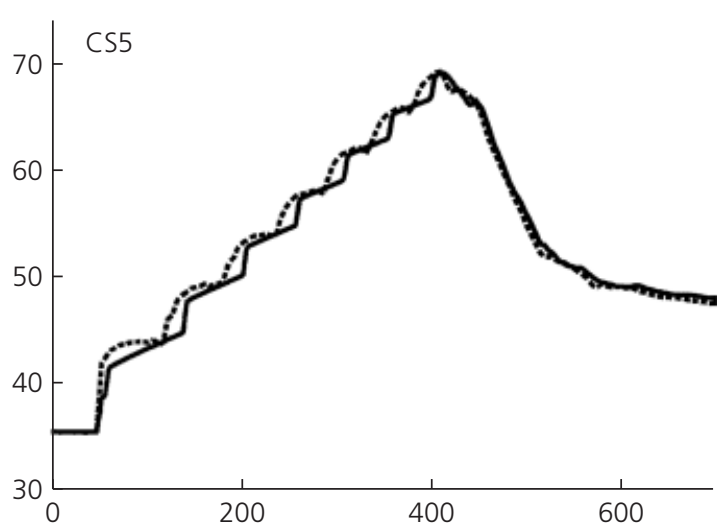

(b)

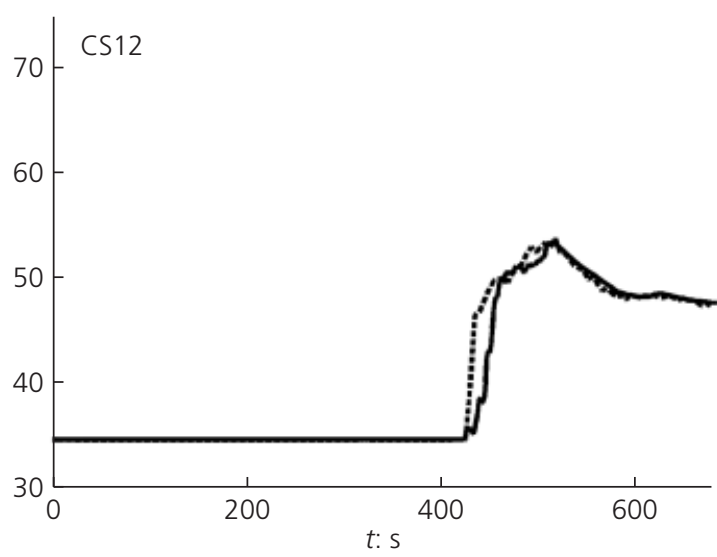

(d)

Figure 2. Stage hydrographs for F-case 11 without initial breach

Overall, the agreement between the computed results and the flume experimental data demonstrates the ability of the model to resolve flooding due to a dam breach composed of nearly uniform sediment with and without an initial breach. The modification coefficient $\phi=4.0$ in Equation $6 \mathrm{~b}$ is adopted in the present modelling, which is appreciably different from $\phi=6.0$ used by Yue (2010) and Cao et al. (2011b). This is because the effect of hindered sediment settling on the deposition flux is considered in the present model, while it was neglected in the previous works. Also, the minimum water depth adopted to distinguish between the wetting and drying beds in the present work $\left(1.0 \times 10^{-6} \mathrm{~m}\right)$ differs from that $\left(1.0 \times 10^{-3} \mathrm{~m}\right)$ used by Yue (2010) and Cao et al. (2011b). As illustrated by the computational tests described in this paper, it can be concluded that the present model can appropriately deal with irregular terrain, can handle the wettingdrying transition and can model the breach of dams composed of nearly uniform sediment.

\section{Case study}

The Tangjiashan landslide dam was the largest generated by the $M_{\mathrm{S}} 8.0$ Wenchuan earthquake. The dam heights were about $90 \mathrm{~m}$ and $120 \mathrm{~m}$ on the right- and left-hand sides of the river channel respectively. The elevation of the dam top was about $750 \mathrm{~m}$ above datum. The length of the dam along the river was $803 \mathrm{~m}$ and the width of the dam across the river was $611 \mathrm{~m}$. Shortly after the earthquake, a diversion channel was excavated to mitigate the potential flood hazard. This channel initially had a trapezoidal cross-section, was $475 \mathrm{~m}$ in length, $13 \mathrm{~m}$ deep and $8 \mathrm{~m}$ wide at the bottom (Liu et al., 2010). The sediment size of the dam material ranged widely from $4.9 \mathrm{~mm}$ to $710 \mathrm{~mm}$ vertically (Table 1), with a medium diameter of $8 \mathrm{~mm}$. These data were derived from two available samples (Chang and Zhang, 2010; Liu et al., 2010). The difference in diameter in Liu et al. (2010) and Chang and Zhang (2010) arose from the method and location of sampling in the field. The sediment size distribution in the vertical, as listed in Table 1, is used for calculating bed sediment entrainment flux within the domain initially occupied by the landslide dam. Linear interpolation is used to calculate the size at specific elevations along the vertical by virtue of the limited available data. Beyond the domain of the initial dam, a fixed sediment size is used, roughly estimated to be $10 \mathrm{~mm}$ based on the sediment size above elevation $729 \mathrm{~m}$ (Table 1), because there was no measured data of the river bed composition. A series of computational tests using differing fixed sediment size for the region beyond the initial dam 


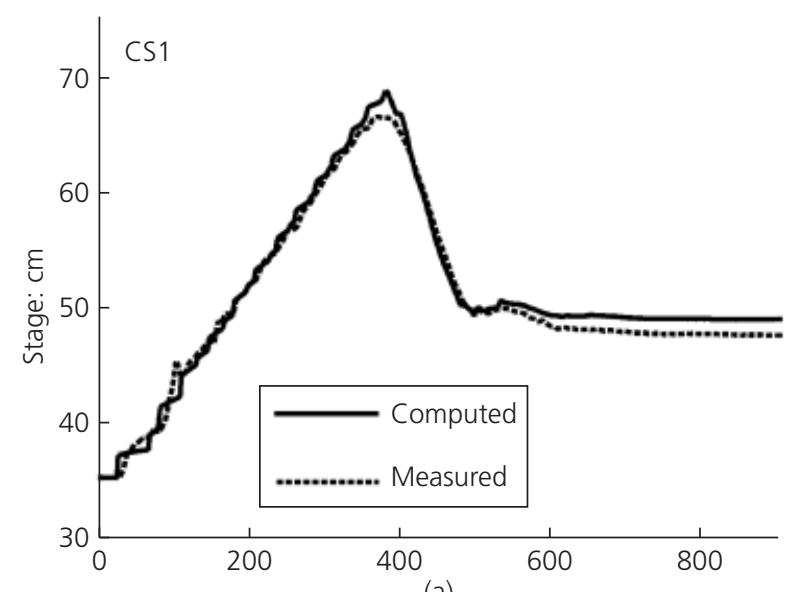

(a)

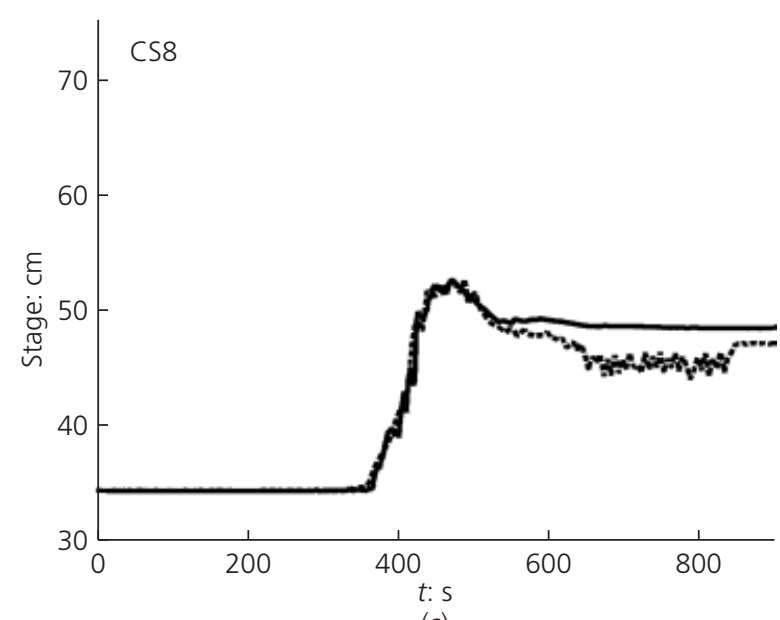

(c)

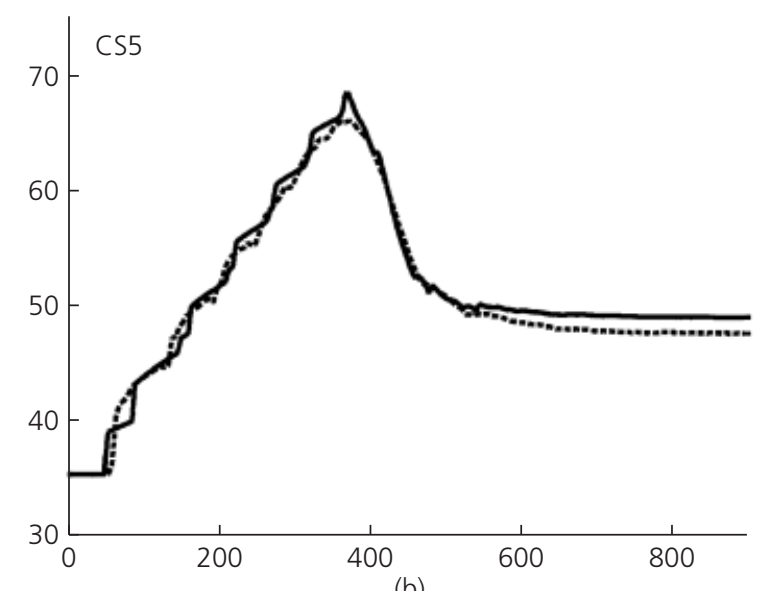

(b)

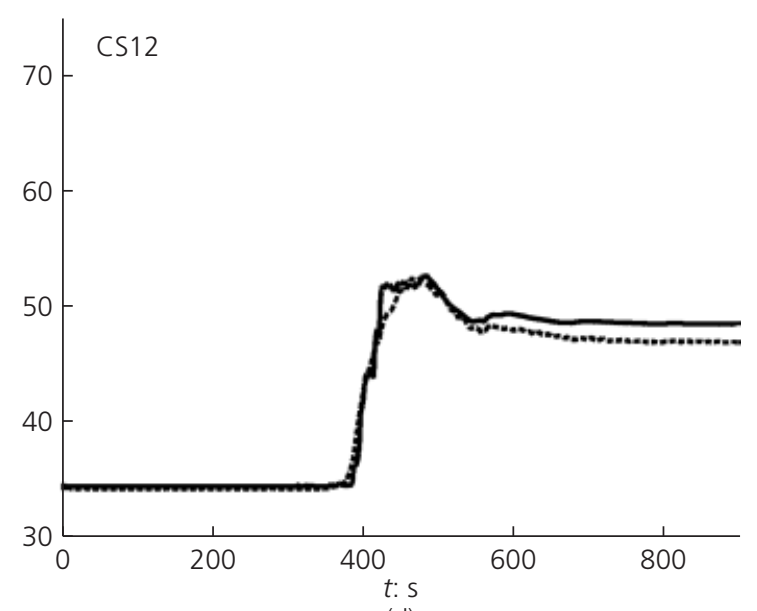

(d)

Figure 3. Stage hydrographs for S-case 7 with initial breach

domain, ranging from $5 \mathrm{~mm}$ to $20 \mathrm{~mm}$, shows that its effect on the dam breach and the flood is negligible.

\subsection{Initial and boundary conditions}

At $6.00 \mathrm{am}$ on 10 June 2008 , which corresponds to $t=0 \mathrm{~h}$ in the present modelling (i.e. the initial condition), the water stage in the dammed lake is set at $742.8 \mathrm{~m}$ and the stage downstream of the dam is set to $665.0 \mathrm{~m}$. At this moment, the barrier lake and the downstream river channel are assumed to be at rest. At the upstream boundary, a discharge of $80.0 \mathrm{~m}^{3} / \mathrm{s}$ is specified. To determine the stage at the upstream boundary, a stage-water volume relation for the dammed lake is introduced in which the inflow at the upstream boundary and outflow at the breach of the dam are taken into account. Once the water volume is calculated after each time step, the stage at the upstream boundary is obtained. Thus, the velocity distribution at the upstream boundary can be approximated using local flow depths across the channel. At the downstream boundary, an open boundary condition is assumed as no measured data are available.

\subsection{Results and sensitivity analysis}

The present study uses Manning's $n=0 \cdot 035$, a modification coefficient for entrainment $\phi=3.0$ (slightly smaller than $\phi=4.0$ for flume experiments, as shown in Figures 2 and 3), a value of $S_{\mathrm{R}}=0.40$ related to the repose angle of the sediment and a critical side slope $S_{\mathrm{c}}=0 \cdot 8$. This set of parameters is referred to as the 'standard' parameters. In the sensitivity analysis, adjusted values are used for these parameters, as detailed below.

Figure 5 shows a comparison of the observed and computed discharge hydrograph (Figure 5(a)), stage hydrograph (Figure $5(\mathrm{~b})$ ), bed deformation (Figure 5(c)) and evolution of water surface width of the breach (Figure 5(d)). In general, the computed results using the standard parameters specified above agree with the observed data rather well. The discharge hydrograph (Figure 5(a)) is nearly in accordance with the observed data. The stage hydrograph (Figure 5(b)) is slightly lower than the observed data before scouring terminated and marginally higher than the observation afterwards. The bed deformation 
Coupled modelling of flood due to

natural landslide dam breach

Huang, Cao, Yue, Pender and Zhou

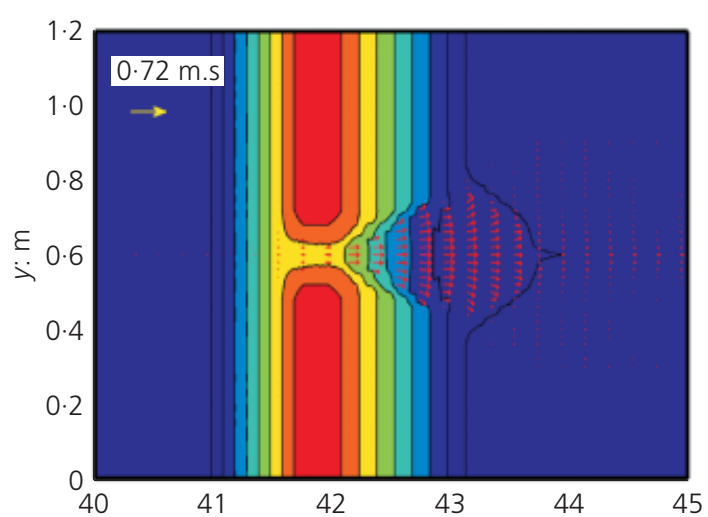

(a)

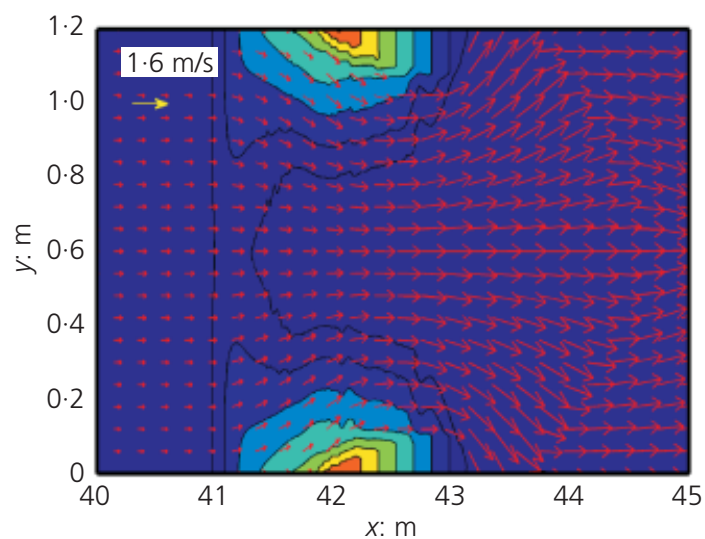

(c)

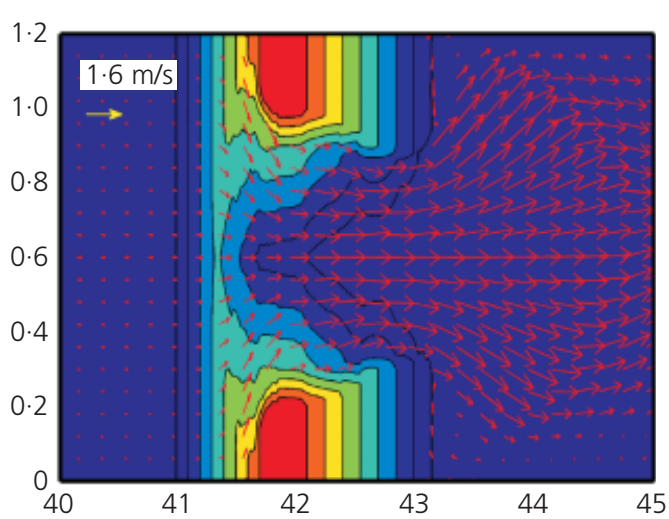

(b)

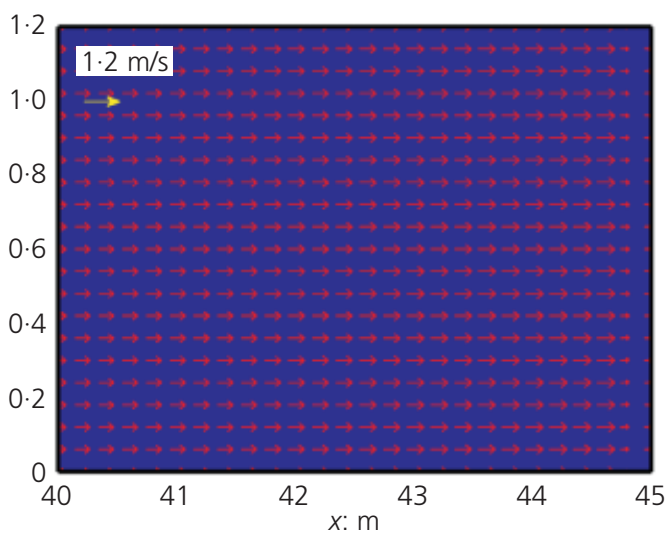

(d)

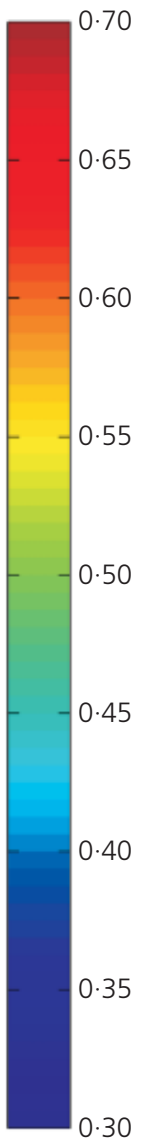

30

Figure 4. Breach development and velocity distribution for S-case 7 (bed elevation in metres): (a) $t=280 \mathrm{~s}$; (b) $t=380 \mathrm{~s}$; (c) $t=400 \mathrm{~s}$; (d) $t=600 \mathrm{~s}$

\begin{tabular}{lcc}
\hline Elevation: $m$ & \multicolumn{2}{c}{ Medium diameter: $\mathrm{mm}$} \\
\cline { 2 - 3 } & Sample 1 & Sample 2 \\
\hline 740 & $11 \cdot 0$ & $4 \cdot 9$ \\
730 & $9 \cdot 0$ & $8 \cdot 0$ \\
729 & $24 \cdot 0$ & $26 \cdot 0$ \\
700 & $710 \cdot 0$ & $660 \cdot 0$ \\
& \\
Table 1. Sediment size distribution along depth. Based on the \\
grain size distribution curve for sediments (Chang and Zhang, \\
2010)
\end{tabular}

process (Figure 5(c)) agrees with the observed data fairly well. The evolution of the water surface width of the breach (Figure $5(\mathrm{~d}))$ is in reasonable agreement with that observed before $t=8 \mathrm{~h}$, but deviates considerably from the measured thereafter. The maximum water surface width from the modelling is $138.6 \mathrm{~m}$, which compares with $145 \mathrm{~m}$ from the observed data. According to Liu et al. (2010), the water surface width deceased rapidly as the stage of the reservoir decreased. Unfortunately, it is not clear why the water surface width did not change from $t=8 \mathrm{~h}$ to $t=13 \mathrm{~h}$ when the water stage decreased rapidly (Figure $5(d)$ ). It is difficult to unravel why the computed water surface width deviates so much from that observed at $t=8-13 \mathrm{~h}$ without more detailed information on the field measurements.

Figure 6 shows the bed topography and velocity distribution of the dam breach. At $t=1.67 \mathrm{~h}$, the downstream surface of the dam is eroded while the breach channel is hardly widened. The river channel downstream the dam sees deposition of sediment scoured from the breach. At $t=5 \mathrm{~h}$, the intense scouring reaches the dam breach. The channel is significantly widened and deepened. After $t=10 \mathrm{~h}$, the widening and deepening of the channel almost terminate because the diameter of the bed material is now too large to erode under the flow conditions. Generally, the velocities are larger in the breach than those in the river channel downstream of the landslide dam. At $t=1.67 \mathrm{~h}$, the larger velocities distribute within the breach. At $t=5 \mathrm{~h}$, the discharge from the breach is larger than that at $t=1.67 \mathrm{~h}$, so the velocities are distributed over a much wider domain. At $t=10 \mathrm{~h}$, 


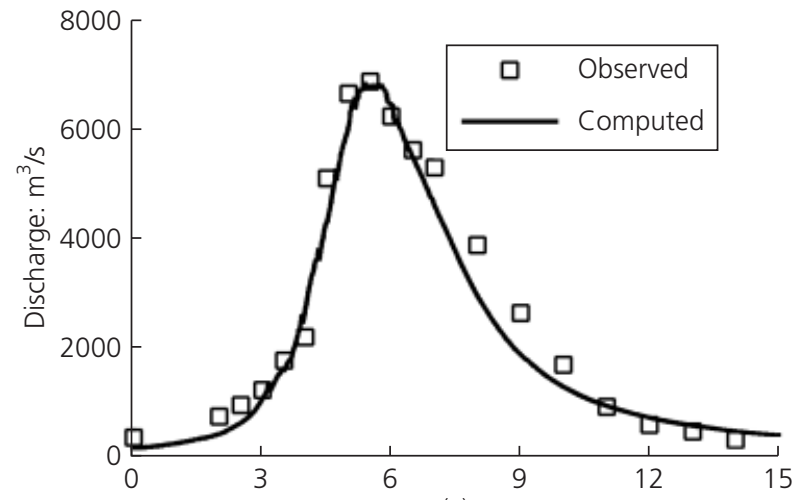

(a)

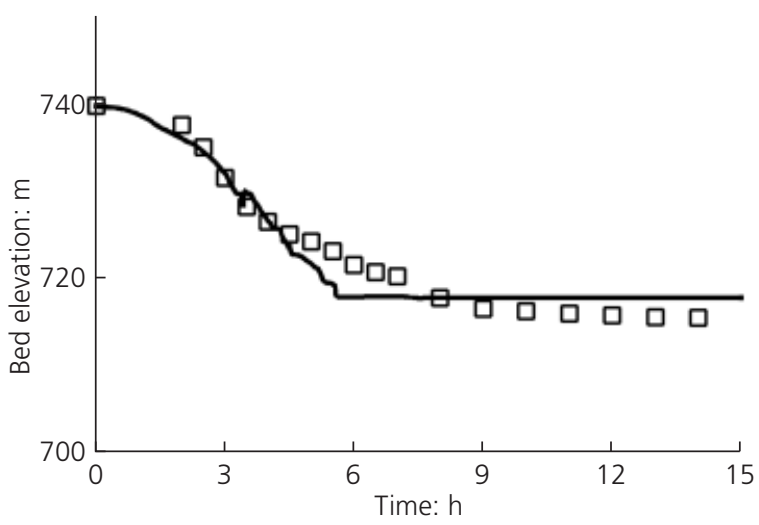

(c)

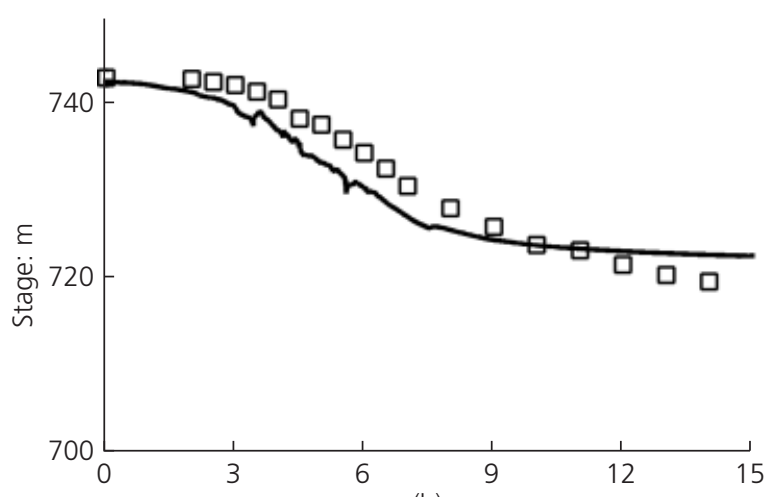

(b)

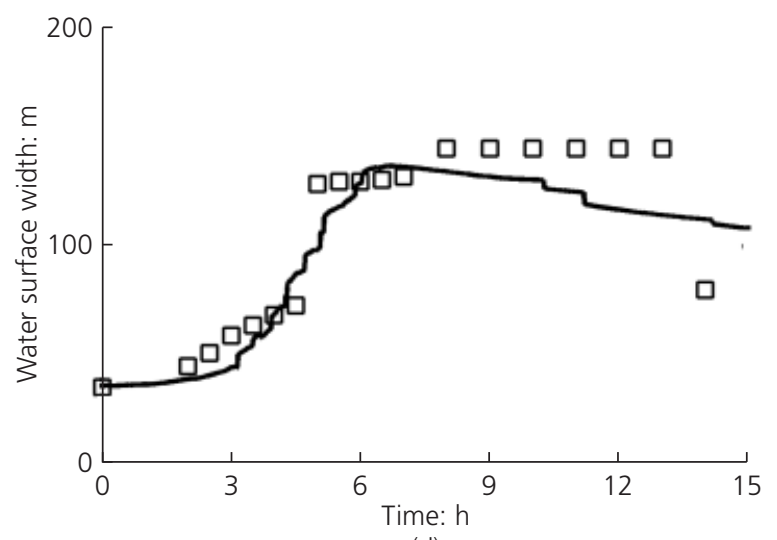

(d)

Figure 5. Flood hydrographs and breach evolution: (a) discharge hydrograph, (b) stage hydrograph, (c) bed elevation, and (d) water surface width in the breach channel

the velocity distribution is confined in a small zone that corresponds to the formation of a new river channel downstream the dam and a smaller discharge from the breach.

While the overall agreement between the computed results and the observed data is fairly good, there are still appreciable discrepancies between the computational results and the observations. These can be ascribed to the following factors.

- The $5 \mathrm{~m}$ resolution of the digital elevation model (DEM) data available for the modelling is relatively low, especially in the vertical direction, which is so crude that it can readily overwhelm the discrepancy between the computed bed elevation and the observed.

- Only a few sediment diameters in the vertical direction are available for the landslide dam, which inevitably renders estimation of the variable size distribution uncertain.

- At some locations, protection efforts were made during channel excavation and therefore the breaching process may be slowed down, which, however, is not incorporated in the modelling.

- The compromised approach dealing the heterogeneous sediments comprising the natural dam inevitably gives rise to uncertainty, which clearly highlights the need for more enhanced modelling of graded sediment transport.

- The use of the uncertain estimations of sediment entrainment and deposition fluxes.

- Direct use of the Manning roughness initially derived for steady and uniform flows over fixed beds, while the dam break flood is highly unsteady and non-uniform over mobile beds.

The present variable-size approach can cope with the heterogeneity of the dam material. For comparison, the conventional fixedsize sediment model was also run to simulate the breaching process. While several different sediment sizes were used for the fixed-size model, only the results of $d=20.0 \mathrm{~mm}$ and $50.0 \mathrm{~mm}$ are shown (Figure 7). Figure 7 shows that, in relation to the 'standard parameters', the results of the fixed-size sediment model deviate considerably from the observed data, both in the peak discharge and the time to the peak discharge. When $d=20.0 \mathrm{~mm}$ is used, which is realistic compared to the observed medium diameter above elevation $729 \mathrm{~m}$ (Table 1), the early phase (roughly, $t<5 \mathrm{~h}$ ) of the discharge hydrograph agrees well 


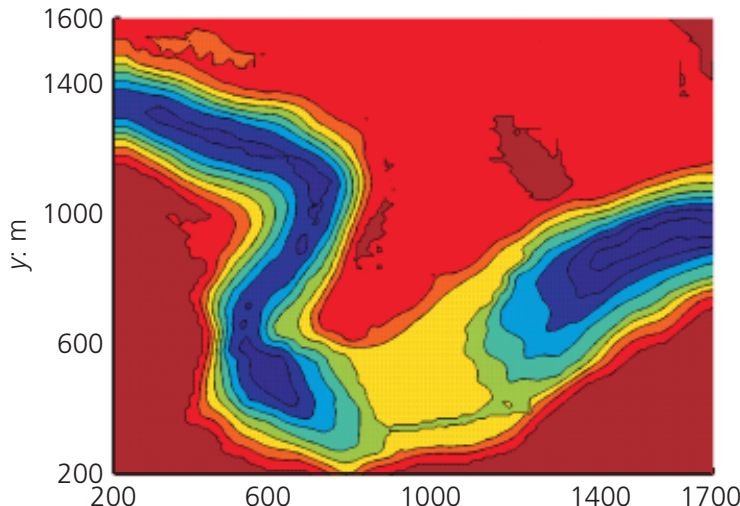

(a)

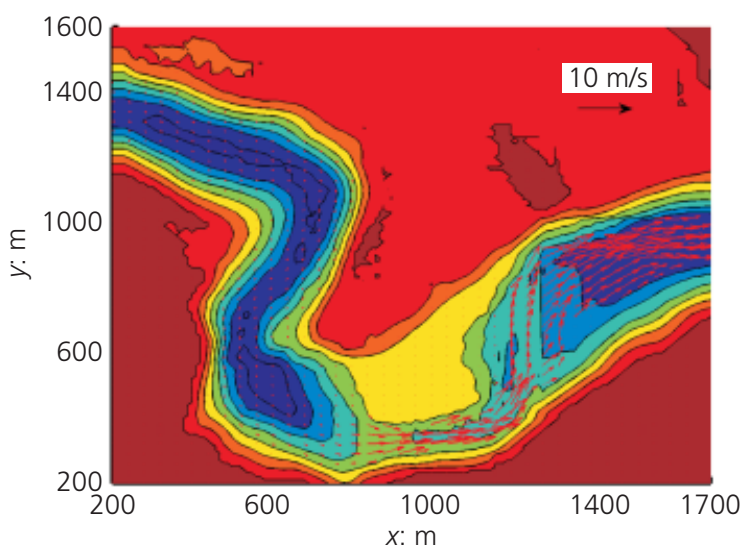

(c)

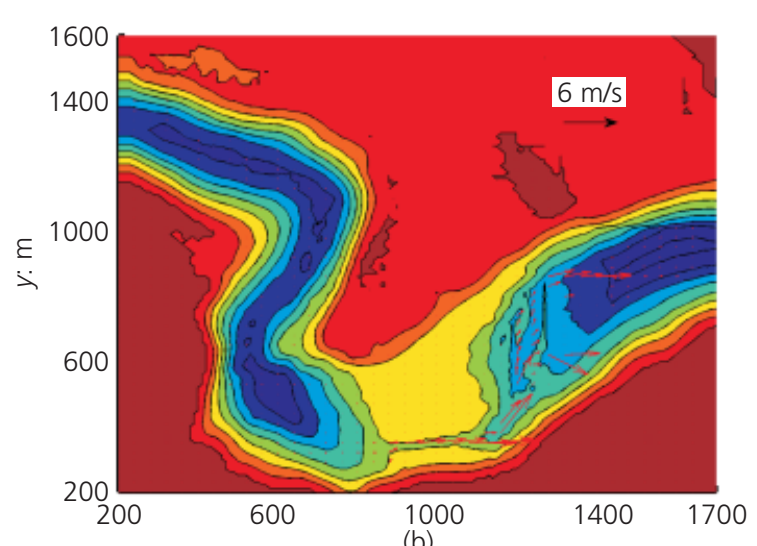

(b)

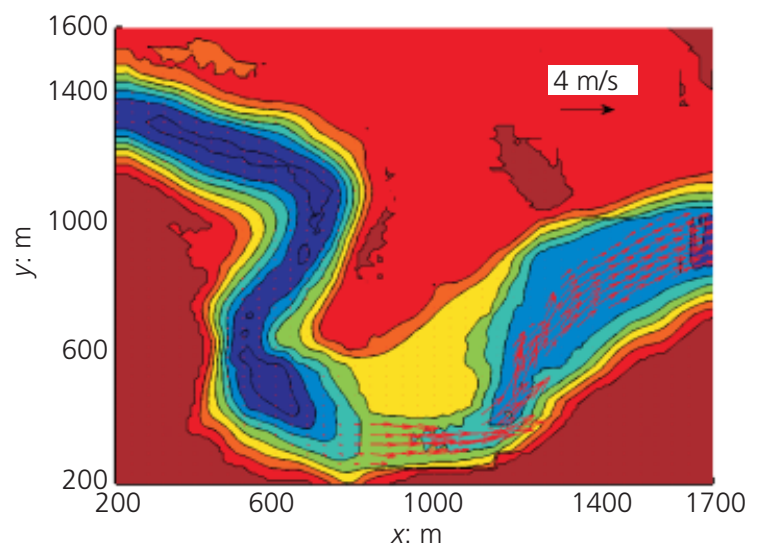

(d)

Figure 6. Velocity distribution over bed topography (bed elevation in metres): (a) $t=0 \mathrm{~h}$; (b) $t=1.67 \mathrm{~h}$; (c) $t=5 \mathrm{~h}$; (d) $t=10 \mathrm{~h}$

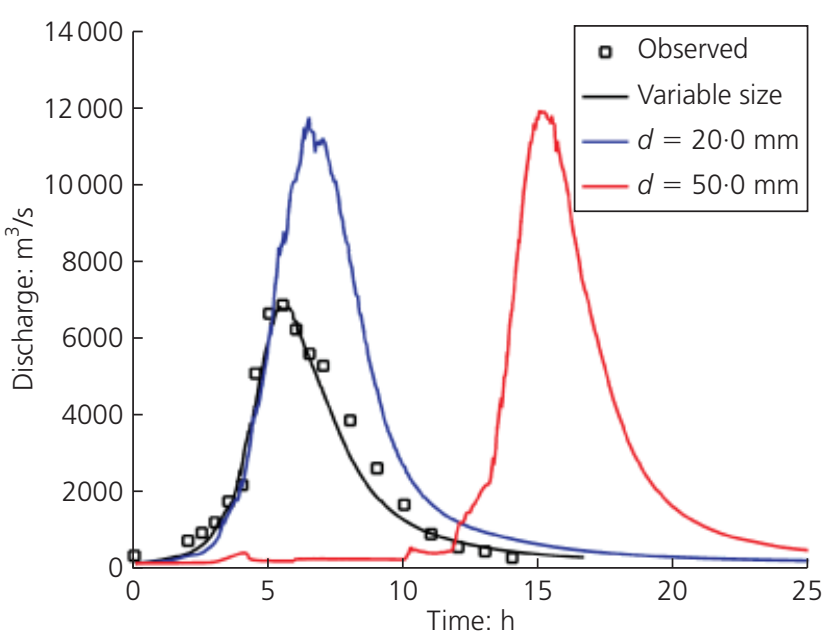

Figure 7. Discharge hydrographs based on the variable-size approach and fixed-size model compared with observed data with the field data. Later, the value of $d=20.0 \mathrm{~mm}$ used in the modelling is much smaller than the actual medium diameter from the field observation (Table 1) and easier to erode than the real regime; the computed discharge is thus significantly larger than the observed. The computed peak discharge is nearly $11809 \mathrm{~m}^{3} / \mathrm{s}$ at $t=6.49 \mathrm{~h}$, in comparison with that observed $\left(6900 \mathrm{~m}^{3} / \mathrm{s}\right.$ at $t=5.5 \mathrm{~h}$. If $d=50 \cdot 0 \mathrm{~mm}$ is used, which is much larger than the actual medium diameter above elevation $729 \mathrm{~m}$ (Table 1), the scouring of the breach is significantly deferred. The corresponding discharge hydrograph sees a $10 \mathrm{~h}$ delay approximately in the time to peak discharge compared with that observed. Yet the computed peak discharge using $d=50.0 \mathrm{~mm}$ differs little from that using $d=20.0 \mathrm{~mm}$. This leads one to speculate that both values of sediment diameter $(20.0$ and $50.0 \mathrm{~mm})$ used in the fixed-size sediment model are so small in comparison with the real situation below elevation $729 \mathrm{~m}$ (as evidenced by observed data at elevation $700 \mathrm{~m}$ (Table 1)) that the impact of the difference in sediment diameter on the flood is negligible. Therefore, it is concluded that sediment particle size plays a paramount 
role in landslide dam breach and the resulting flood. Physically, this is determined by the fact that sediments comprising a natural landslide dam are widely graded and cannot be properly represented by a fixed size. Consequently, it is critical to properly account for the high heterogeneity of the material of natural landslide dams if the modelling is to reasonably resolve the dam breach development and the resulting flood. As a first step towards a solution to this problem, the present 'compromised' approach for heterogeneous sediments can reproduce reasonable results in comparison with available observations and therefore shows great potential. However, the model parameters would have to be carefully estimated according to experience to be accrued from more field cases. There is no doubt that a complete graded sediment model would offer greater potential for refined modelling of a natural landslide dam breach along with the resulting flood.

It is interesting to identify the extent to which the modelling results from the variable-size approach are sensitive to the parameters. Three parameters were tuned, based on the standard values: Manning's roughness $n$, the modification coefficient for entrainment $\phi$ and the critical side slope $S_{\mathrm{c}}$. Figure 8(a) shows the impact of $n$ on the discharge hydrograph. With increased $n$, the peak discharge increases and its occurrence is considerably advanced in time. Specifically, with $n$ varying from 0.03 to 0.04 , the peak discharge increases from $4705.8 \mathrm{~m}^{3} / \mathrm{s}$ to $9129.6 \mathrm{~m}^{3} / \mathrm{s}$ and its timing is advanced from $t=8.45 \mathrm{~h}$ to $t=4.61 \mathrm{~h}$. Figure 8 (b) illustrates the effect of $\phi$, which is involved in estimating the sediment entrainment flux. With a larger value of $\phi$, the entrainment flux becomes larger under the same flow conditions, which corresponds to faster scouring of the dam and accordingly a raised peak discharge. At $\phi=2.5$ the peak discharge is $5731.7 \mathrm{~m}^{3} / \mathrm{s}$ and its occurrence is at $t=7.03 \mathrm{~h}$, while at $\phi=3.5$ the peak discharge is $7552.9 \mathrm{~m}^{3} / \mathrm{s}$ and occurs at $t=5.53 \mathrm{~h}$. Figure 8(c) shows the role of the critical side slope in modulating the flood. With a larger critical side slope, the peak discharge increases appreciably and the timing to peak discharge is advanced. The peak discharge is $6219.6 \mathrm{~m}^{3} / \mathrm{s}$ at $t=6.54 \mathrm{~h}$ for $S_{\mathrm{c}}=0.6$, while the peak discharge is $6977.7 \mathrm{~m}^{3} / \mathrm{s}$ and its timing is advanced to $t=5.03 \mathrm{~h}$ for $S_{\mathrm{c}}=1.0$.

Attempts have been made to reconcile the computed discharge hydrograph from the fixed-size model with the observed data by tuning $n, \phi$ and $S_{\mathrm{c}}$. More than 40 combinations of the parameters were tried within the ranges $n \in[0 \cdot 015,0 \cdot 05], \phi \in[1 \cdot 0,5 \cdot 0]$ and $S_{\mathrm{c}} \in[0 \cdot 6,2 \cdot 0]$. The results of 14 combinations are presented in Figures 9 and 10 to show the impacts of the parameters on the discharge hydrograph. Qualitatively, the impacts of the parameters (Figures 9 and 10) are similar to those for the variablesize model (Figure 8). Briefly, with a larger value of $n, \phi$ or $S_{\mathrm{c}}$,

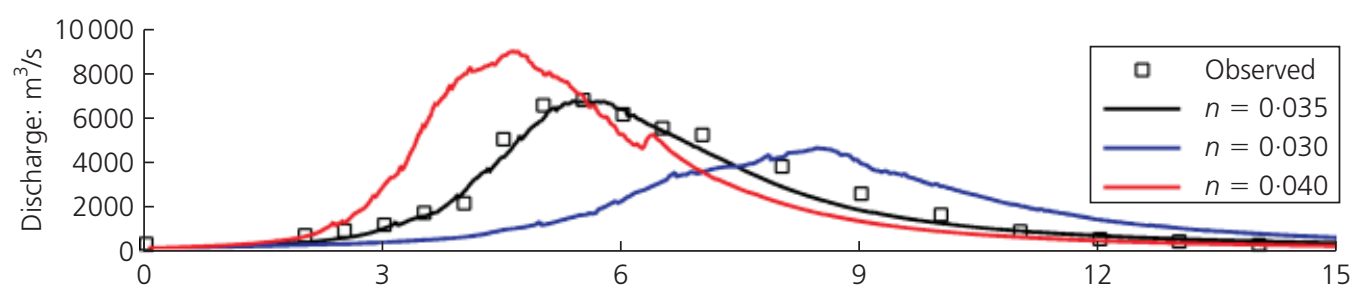

(a)

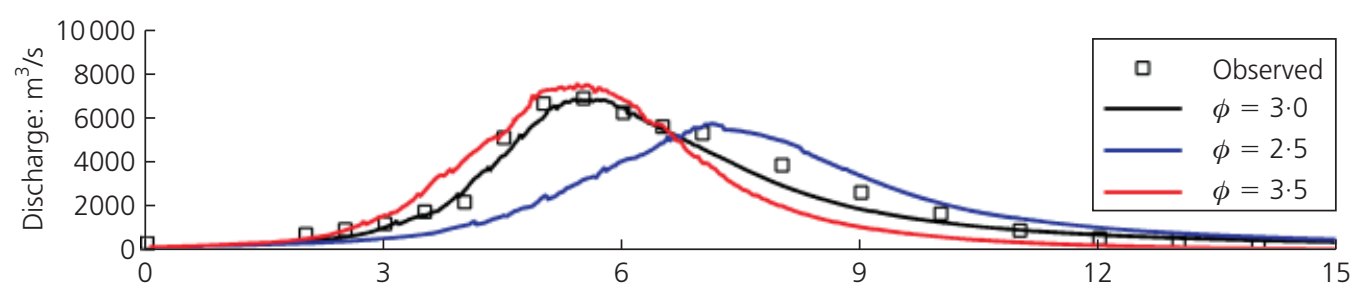

(b)

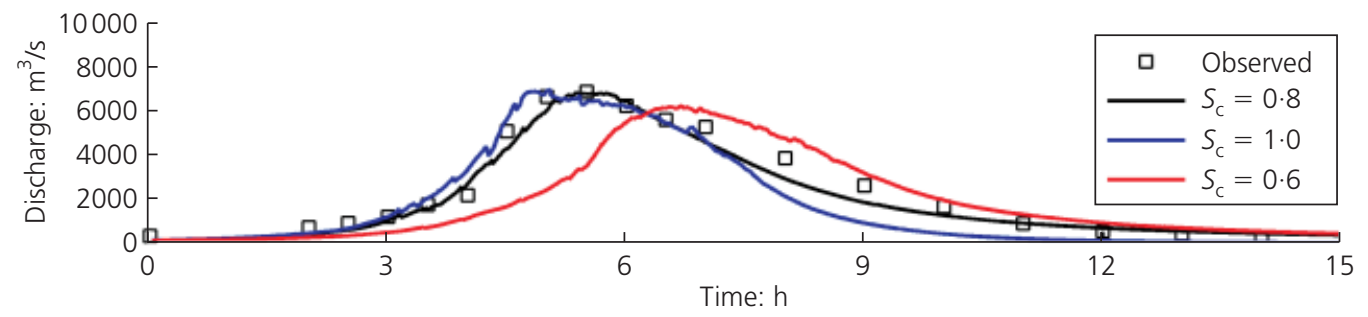

(c)

Figure 8. Impacts of (a) Manning's roughness $n$, (b) the modification coefficient for entrainment $\phi$ and (c) critical slope $S_{c}$ on discharge hydrograph based on the variable-size model 


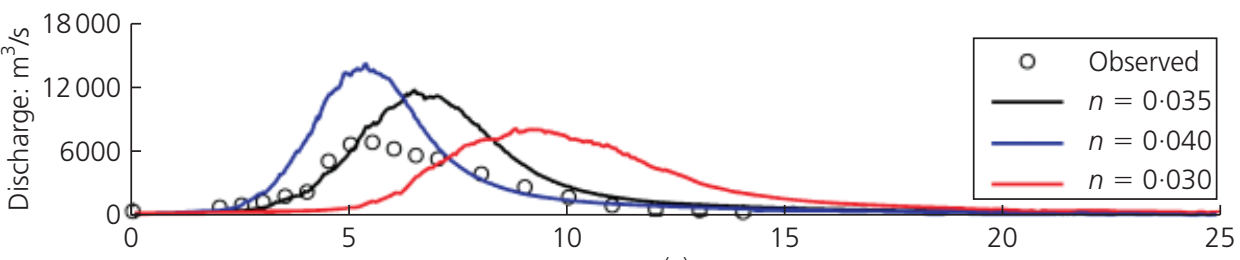

(a)

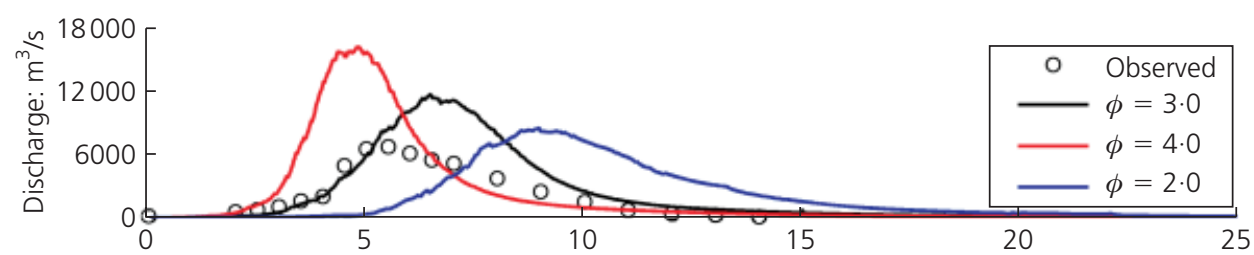

(b)

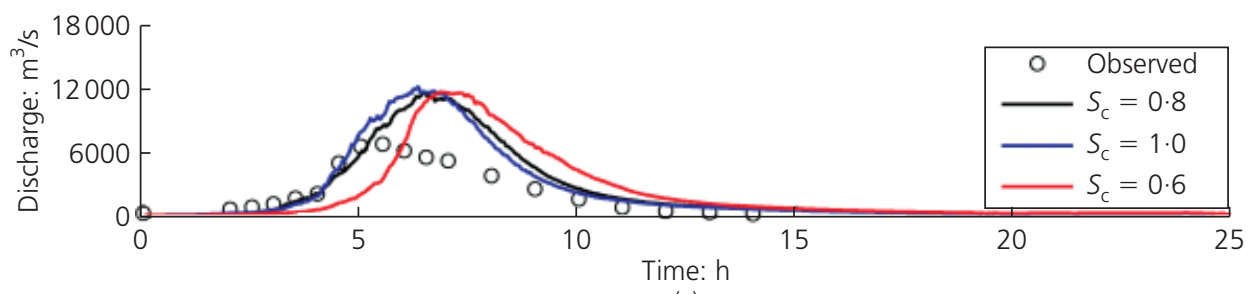

(c)

Figure 9. Impacts of (a) Manning's roughness $n$, (b) the modification coefficient for entrainment $\phi$ and (c) critical slope $S_{c}$ on discharge hydrograph based on the fixed-size model with $d=20 \cdot 0 \mathrm{~mm}$

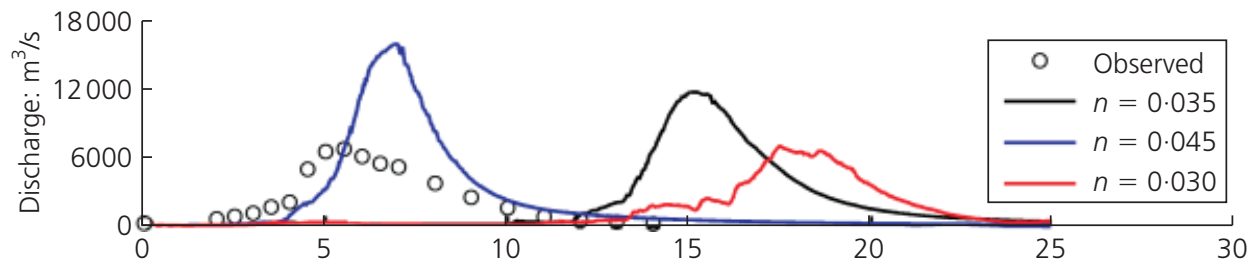

(a)

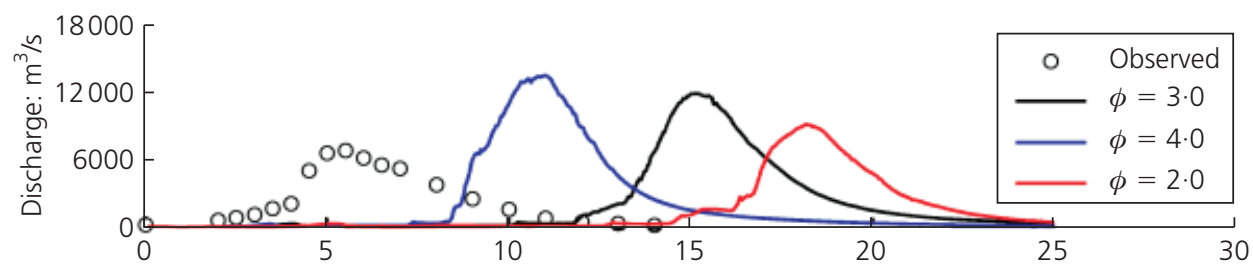

(b)

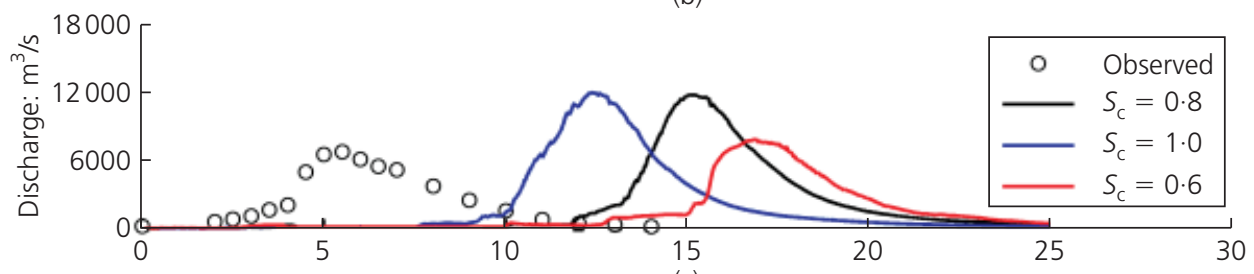

(c)

Figure 10. Impacts of (a) Manning's roughness $n$, (b) the modification coefficient for entrainment $\phi$ and (c) critical slope $S_{c}$ on discharge hydrograph based on the fixed-size model with $d=50.0 \mathrm{~mm}$ 
the peak discharge is higher and its timing is advanced (Figures 9 and 10), and vice versa. Overall, the computational exercises indicate that the computed discharge hydrograph from the fixedsize model and the observed data cannot be reconciled by tuning the parameters within wide ranges (i.e. those related to sediment diameter, entrainment flux, critical side slope and Manning roughness). It is suggested, therefore, that the heterogeneity of natural landslide dam material is essential and must be accounted for in the modelling. The discharge hydrographs from previous studies seem to agree with the observed data (e.g. Mori et al., 2010; Wang et al., 2008a, 2008b). Most likely, the substantial assumptions and approximations used in the formulations of previous studies have cancelled out the effects of the heterogeneity of the material of a natural landslide dam.

Because there are no observed hydrograph data available for specifying the downstream boundary condition, an open boundary condition was assumed for all the modelling cases above. Here, it is compared against the local uniform flow boundary condition based on the variable-size approach. Figure 11 shows that different boundary conditions may result in different breaching pro-

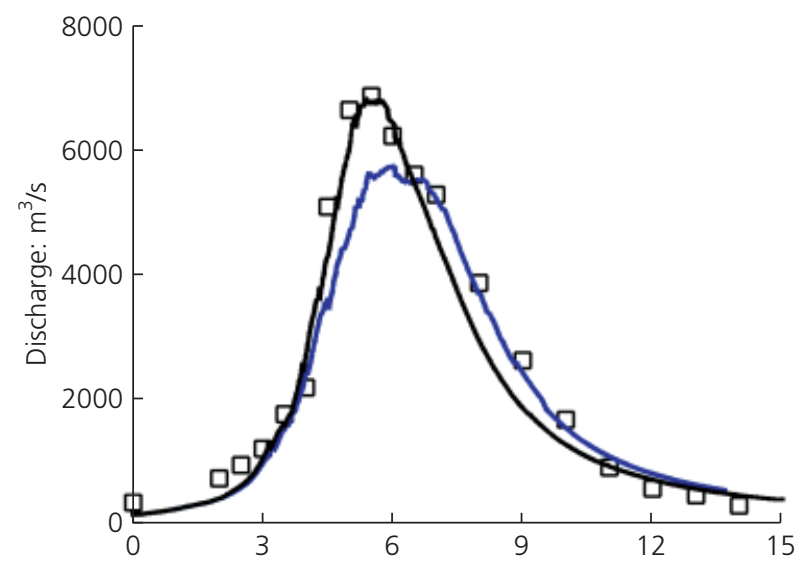

(a)

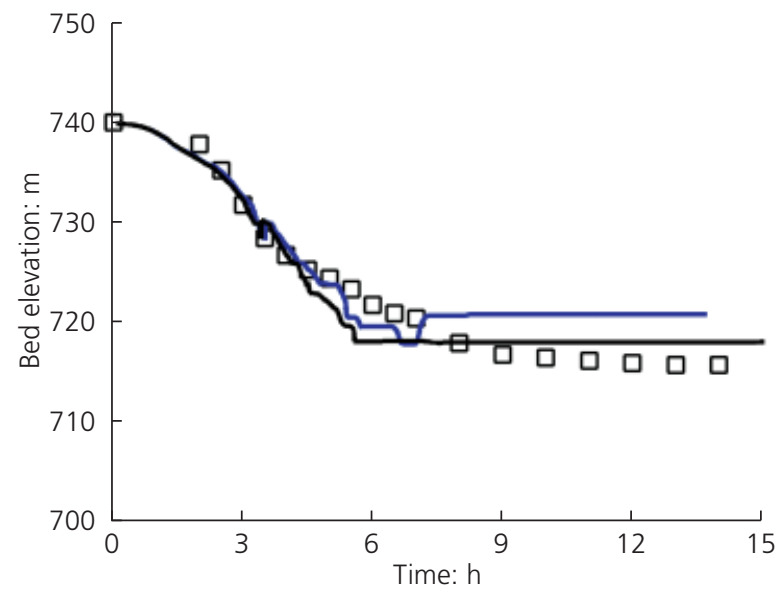

(c) cesses. The open boundary condition can give a fairly good agreement between the computed and observed breaching process, while the local uniform flow condition leads to an appreciable difference. Mainly, the stage and bed elevation modelling in line with the local uniform flow condition are appreciably higher than their counterparts using the open boundary condition, while the peak discharge is lowered and deferred slightly in time, and the water surface width is narrowed.

\section{Conclusions}

A coupled 2D physically enhanced model has been developed to solve for natural landslide dam failure and the resulting flood. This model can effectively deal with the drying-wetting transition and complex terrain because of the well-balanced numerical algorithm implemented to solve the governing equations. A compromised approach is introduced to cope with the heterogeneity of the sediments comprising natural landslide dams. As applied to the case of Tangjiashan landslide dam, the modelled stage and discharge hydrographs, as well as the widening and deepening of the breach, agree with the observed data rather well. In contrast, a fixed-size sediment model fails, even if the model

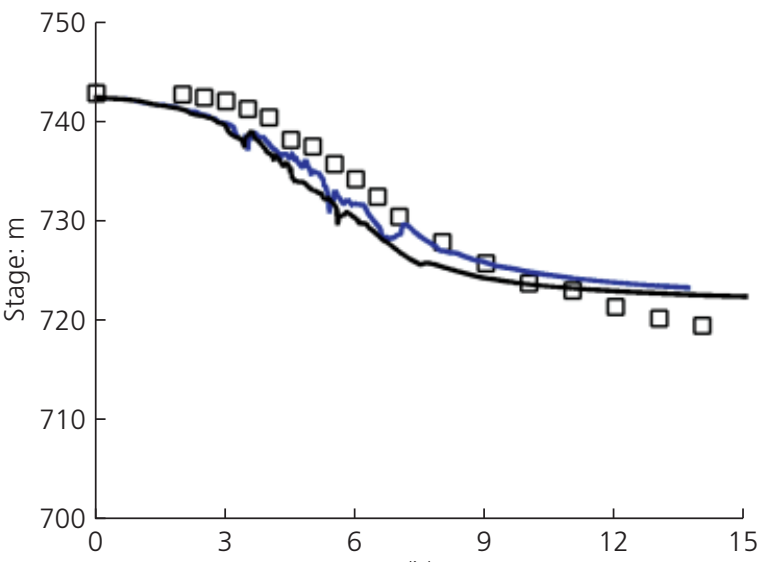

(b)

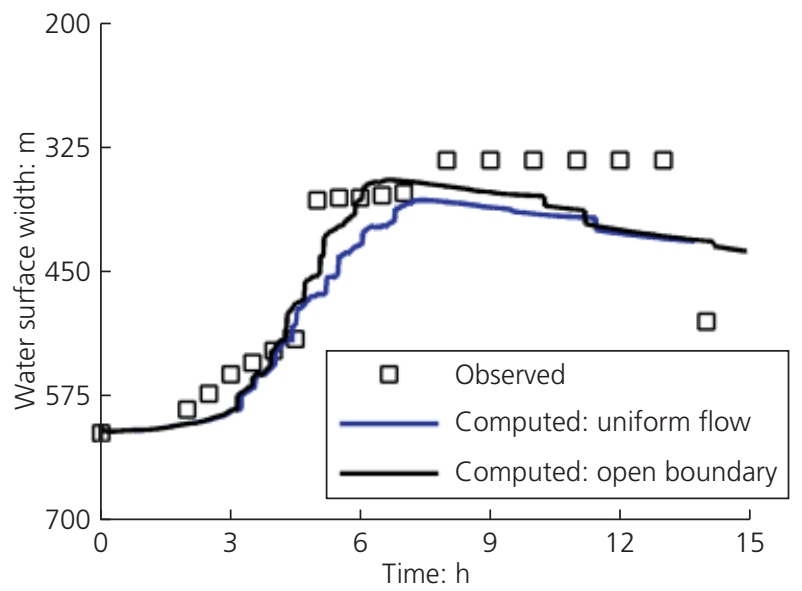

(d)

Figure 11. Impact of downstream boundary conditions on discharge hydrograph from the variable-size model 
parameters are considerably adjusted. The role of the heterogeneity of the natural landslide dam material is critical in dictating the breaching process and the resulting flood. It certainly merits careful consideration in future studies and a complete graded sediment model is warranted.

The major limitation of the present case study of Tangjiashan landslide dam arises from the low resolution of the DEM data available and the limited availability of data for the composition of the natural dam. The closure formulations for boundary resistance and sediment entrainment bear empiricism to a certain extent, and are the primary sources of model uncertainty. This clearly points to the need for fundamental investigations of the interactions of unsteady and rapidly varying turbulent flows, graded sediments and irregular mobile beds

\section{Acknowledgements}

This work is funded by National Key Basic Research and Development Program (973 Program) of China (grant 2007CB714106) and the Natural Science Foundation of China (grants 10932012 and 11172217). Special thanks are due to Dr D. Y. Zhong for supplying the observed field data and for his constructive suggestions.

\section{REFERENCES}

ASCE/EWRI (ASCE/EWRI Task Committee on Dam/Levee Breaching) (2011) Earthen embankment breaching. Journal of Hydraulic Engineering ASCE 137(12): 1549-1564.

Baker V, Benito G and Rudoy AN (1993) Paleohydrology of late pleistocene superflooding, Altay mountains, Siberia. Science 259(5093): 348-350.

Brufau P, Vázquez-Cendón ME and García-Navarro P (2002) A numerical model for the flooding and drying of irregular domains. International Journal for Numerical Methods in Fluids 39(3): 247-275.

Cao Z, Pender G, Wallis S and Carling P (2004) Computational dam-break hydraulics over erodible sediment bed. Journal of Hydraulic Engineering ASCE 130(7): 689-703.

Cao Z, Yue Z and Pender G (2011a) Landslide dam failure and flood hydraulics. Part I: experimental investigation. Natural Hazards 59(2): 1003-1019.

Cao Z, Yue Z and Pender G (2011b) Landslide dam failure and flood hydraulics. Part II: coupled mathematical modelling. Natural Hazards 59(2): 1021-1045.

Cao Z, Yue Z and Pender G (2011c) Flood hydraulics due to cascade landslide dam failure. Journal of Flood Risk Management 4(2): 104-114.

Cencetti C, Fredduzzi A, Marchesini I, Naccini M and Tacconi P (2006) Some considerations about the simulation of breach channel erosion on landslide dams. Computational and Geosciences 10(2): 201-219.

Chang DS and Zhang LM (2010) Simulation of the erosion process of landslide dams due to overtopping considering variations in soil erodibility along depth. Natural Hazards and Earth System Science 10(4): 933-946.
Chen YJ, Zhou F, Feng Y and Xia YC (1992) Breach of a naturally embanked dam on Yalong River. Canadian Journal of Civil Engineering 19(5): 811-818.

Costa JE and Schuster RL (1988) The formation and failure of natural dams. Geological Society of America Bulletin 100(7): 1054-1068.

Cruden DM, Lu ZY and Thomson S (1997) The 1939 Montagneuse River landslide, Alberta. Canadian Geotechnical Journal 34(5): 799-810.

Cui P, Zhu Y, Han Y, Chen X and Zhuang J (2009) The 12 May Wenchuan earthquake-induced landslide lakes: distribution and preliminary risk evaluation. Landslides 6(3): 209-223.

Dai FC, Lee CF, Deng JH and Tham LG (2005) The 1786 earthquake-triggered landslide dam and subsequent dambreak flood on the Dadu River, southwestern China. Geomorphology 65(3-4): 205-221.

Hirsch C (1990) Numerical Computation of Internal and External Flows. Volume 2: Computational Methods for Inviscid and Viscous Flows. Wiley, London, UK.

Korup K (2002) Recent research on landslide dams-a literature review with special attention to New Zealand. Progress in Physical Geography 26(2): 206-235.

Liang QH and Marche F (2009) Numerical resolution of wellbalanced shallow water equations with complex source terms. Advances in Water Resources 32(6): 873-884.

Liu N, Chen ZY, Zhang JX, Lin W, Chen WY and Xu WJ (2010) Draining the Tangjiashan barrier lake. Journal of Hydraulic Engineering ASCE 136(11): 914-923.

Meyer-Peter E and Müller R (1948) Formulas for bed-load transport. Proceedings of the 2nd Meeting of the International Association for Hydraulic Research. IAHR, Stockholm, Sweden, pp. 39-64.

Mori T, Sakaguchi T, Sawa Y, Mizuyama T, Satofuka Y, Ogawa K, Usuki N and Yoshino K (2010) Method of estimation for flood discharges caused by overflow erosion of landslide dams and its application in as a countermeasure. Proceedings of International Symposium on Disaster Mitigation of Debris Flows, Slope Failures and Landslides, Taipei, pp. 293-301.

Richardson J and Zaki W (1954) Sedimentation and Fluidisation: Part 1. Transactions of the Institution of Chemical Engineers 32: $35-53$.

Shang YJ, Yang ZF, Li LH, Liu DA, Liao QL and Wang YC (2003) A super-large landslide in Tibet in 2000: background, occurrence, disaster and origin. Geomorphology 54(3-4): $225-243$.

Toro EF (2001) Shock-capturing Methods for Free-surface Shallow Flows. Wiley, London, UK.

Wang GQ, Liu F, Fu XD and Li T (2008a) Simulation of dam breach development for emergency treatment of the Tangjiashan Quake Lake in China. Science in China, Series E: Technological Sciences 51(2): 82-94.

Wang GQ, Zhong DY, Zhang HW, Sun QC and Hu DC (2008b) Numerical simulation of water release of Tangjiashen barrier lake formed in Wenchuan earthquake. Chinese Science Bulletin 53(24): 3127-3133 (in Chinese). 
Wang ZG and Bowles DS (2006a) Three-dimensional noncohesive earthen dam breach model. Part 1: theory and methodology. Advances in Water Resources 29(10): $1528-$ 1545 .

Wang ZG and Bowles DS (2006b) Three-dimensional noncohesive earthen dam breach model. Part 2: validation and applications. Advances in Water Resources 29(10): 14901503.

Wu WM (2007) Computational River Dynamics. Taylor \& Francis, London, UK.
Wu WM, Wang SSY and Jia YF (2000) Nonuniform sediment transport in alluvial rivers. Journal of Hydraulic Research 38(6): 427-434.

Yue ZY (2010) Mechanism and Hydrodynamic Processes of Natural Dam Failure - Experiments and Coupled Mathematical Modelling. PhD thesis, Wuhan University, Wuhan, Hubei, China (in Chinese).

Zhang RJ and Xie JH (1993) Sedimentation Research in China: Systematic Selections. China Water and Power Press, Beijing, China.

\section{WHAT DO YOU THINK?}

To discuss this paper, please email up to 500 words to the editor at journals@ice.org.uk. Your contribution will be forwarded to the author(s) for a reply and, if considered appropriate by the editorial panel, will be published as a discussion in a future issue of the journal.

Proceedings journals rely entirely on contributions sent in by civil engineering professionals, academics and students. Papers should be 2000-5000 words long (briefing papers should be 1000-2000 words long), with adequate illustrations and references. You can submit your paper online via www.icevirtuallibrary.com/content/journals, where you will also find detailed author guidelines. 